\title{
PSZSPT: A joint Planck and SPT-SZ cluster catalog ${ }^{\star}$
}

\author{
J.-B. Melin ${ }^{1}$, J. G. Bartlett ${ }^{2,3}$, P. Tarrío ${ }^{4,1}$, and G. W. Pratt ${ }^{4,1}$ \\ 1 IRFU, CEA, Université Paris-Saclay, 91191 Gif-sur-Yvette, France \\ e-mail: jean-baptiste.melin@cea.fr \\ 2 Université de Paris, CNRS, Astroparticule et Cosmologie, 75006 Paris, France \\ 3 Jet Propulsion Laboratory, California Institute of Technology, 4800 Oak Grove Drive, Pasadena, California, USA \\ 4 AIM, CEA, CNRS, Université Paris-Saclay, Université Paris Diderot, Sorbonne Paris Cité, 91191 Gif-sur-Yvette, France \\ Received 18 September 2020 / Accepted 11 January 2021
}

\begin{abstract}
We present the first cluster catalog extracted from combined space-based (Planck) and ground-based (South Pole Telescope; SPT-SZ) millimeter data. We developed and applied a matched multi-filter (MMF) capable of dealing with the different transfer functions and resolutions of the two datasets. We verified that it produces results consistent with publications from Planck and SPT collaborations when applied on the datasets individually. We also verified that Planck and SPT-SZ cluster fluxes are consistent with each other. When applied blindly to the combined dataset, the MMF generated a catalog of 419 detections $(S / N>5)$, of which 323 are already part of the SPT-SZ or PSZ2 catalogs; 54 are new SZ detections, which have been identified in other catalogs or surveys; and 42 are new unidentified candidates. The MMF takes advantage of the complementarity of the two datasets, Planck being particularly useful for detecting clusters at a low redshift $(z<0.3)$, while SPT is efficient at finding higher redshift $(z>0.3)$ sources. This work represents a proof of concept that blind cluster extraction can be performed on combined, inhomogeneous millimeter datasets acquired from space and ground. This result is of prime importance for planned ground-based cosmic microwave background (CMB) experiments (e.g., Simons Observatory, CMB-S4) and envisaged CMB space missions (e.g., PICO, Backlight) that will detect hundreds of thousands of clusters in the low mass regime $\left(M_{500} \leqslant 10^{14} M_{\odot}\right)$, for which the various sources of intra-cluster emission (gas, dust, synchrotron) will be of the same order of magnitude and hence require broad ground and space frequency coverage with a comparable spatial resolution for adequate separation.
\end{abstract}

Key words. galaxies: clusters: general - large-scale structure of Universe - catalogs

\section{Introduction}

Galaxy clusters constitute unique objects to study structure formation in the Universe. Lying at the nodes of the cosmic web, their distribution in mass and redshift is sensitive to cosmological parameters (e.g., Allen et al. 2011, and references therein). They also represent ideal laboratories to understand galaxy formation and evolution (e.g., Voit 2005, and references therein). Progressing in both cosmology and astrophysics with galaxy clusters requires advances in two directions: increasing the number of known clusters and better understanding their physics. The multi-frequency view is now mandatory to achieve these goals.

Clusters are detected in the optical via their member galaxies, in the X-ray via Bremsstrahlung emission of the embedded hot ionized gas and, more recently, via the SunyaevZeldovich (SZ, Sunyaev \& Zeldovich 1970, 1972) effect. Progress in detecting numerous new clusters has been made in recent years with the advent of SZ surveys (Staniszewski et al. 2009; Menanteau et al. 2010; Planck Collaboration VIII 2011). The current galaxy cluster catalogs are most often extracted from single experiment data. This is the case for the all-sky Abell (Abell et al. 1989), ROSAT (Böhringer et al. 2000, 2001), and Planck (Planck Collaboration VIII 2011; Planck Collaboration XXIX 2014a; Planck Collaboration XXVII 2016a) catalogs.

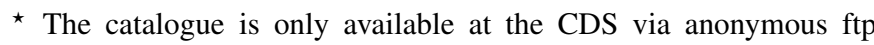
to cdsarc.u-strasbg.fr $(130.79 .128 .5)$ or via http://cdsarc. u-strasbg.fr/viz-bin/cat/J/A+A/647/A106
}

In order to extract clusters with a low signal-to-noise threshold, hybrid methods have been developed recently to clean the single experiment catalogs from spurious detections. These methods have been successfully applied to X-ray (ROSAT) and SZ (Planck) detections using optical (BOSS, DES, WISE, SDSS) data (e.g., Burenin 2017; Finoguenov et al. 2020; Klein et al. 2019). Another approach to extract new clusters is to use data from different frequency bands jointly. The approach was initially proposed by Maturi (2007), but it is difficult to implement in practice because the signals from clusters originate from different physical processes in different frequency bands. It has, nevertheless, been successfully implemented recently by Tarrío et al. $(2016,2018,2019)$ for ROSAT and Planck data.

A final avenue for extracting low signal-to-noise clusters is to combine different observations in the same frequency band. Aghanim et al. (2019), Madhavacheril et al. (2020), and Naess et al. (2020) followed this path and produced, for the first time, combined SZ maps from Planck and ACT data, Planck and ACTPol data, as well as Planck and ACT, ACTPol, and AdvACT data. These works showed, for the first time, the significant gain for cluster science when combining space- and ground-based data in the ACT footprint. Aghanim et al. (2019) also extracted clusters using a match multi-filter (MMF), but they did not publish the associated catalog.

Here, we focus on the Planck and SPT-SZ datasets. We propose a practical implementation of a blind MMF extraction algorithm working on space- and ground-based data jointly, and we publish the associated catalog: PSZSPT. Cross-matches of 
the PSZSPT detections and external catalogs are included. We did not use the Planck and SPT-SZ combined maps proposed by Chown et al. (2018) to produce the PSZSPT catalog because they are not optimized for cluster extraction. We used the SPT maps instead, which are provided in that publication, and we thoroughly tested them against quantities published with the official SPT-SZ catalogs; only then did we combine them with the public Planck maps.

The study presented here is expected to be important for the forthcoming space- and ground-based experiments. The future $\mathrm{CMB}$ experiments will detect low mass clusters $\left(M_{500} \leqslant\right.$ $10^{14} M_{\odot}$ ) for which the SZ signal is expected to be of the same order as the other sources of emission from clusters, in particular the radio and infrared emission of galaxies (e.g., Melin et al. 2018). Combining ground-based $(v<300 \mathrm{GHz})$ and spacebased $(v>300 \mathrm{GHz})$ experiments would help disentangle the various sources of emission [e.g., Simons Observatory (Ade et al. 2019) and Planck or CMB-S4 (Abazajian et al. 2019) and PICO (Hanany, et al. 2019) or Backlight (Basu et al. 2019; Delabrouille et al. 2019)].

We first present the two datasets used in our analysis in Sect. 2. We then recall the characteristics of the MMF in Sect. 3. We apply the MMF on the SPT-SZ and Planck data independently to test the consistency of our results with results published by the two collaborations in Sect. 4. The in-depth work related to Sect. 4 is presented in Appendices A, B, and C. In Sect. 5, we test the consistency between the SPT-SZ and the Planck datasets. We detail the construction of the PSZSPT catalog in Sect. 6 and how we characterize it. We provide a comparison between recovered and published masses in Appendix D. We give the names of the SPT and PSZ2 clusters missed in the PSZSPT catalog in Appendix E. The description of the PSZSPT catalog is given in Appendix F. Finally, we summarize and discuss our findings, and look to future work in Sect. 7.

\section{Data sets}

\subsection{SPT-SZ}

We use the SPT-SZ public maps" "SPT Only Data maps" at 95,150 , and $220 \mathrm{GHz}$. The maps provide a resolution of 1.75 arcmin (full width at half maximum, FWHM), which is slightly degraded with respect to the native resolution of 1.6, 1.1 , and 1.0 arcmin at 95,150 , and $220 \mathrm{GHz}$, respectively (Bleem et al. 2015a). The other key ingredients for the analysis described in this paper are the filter transfer functions, "SPT Filter Transfer Function" at each frequency and the boundary and point source mask "Mask". All the products are provided at Healpix $N_{\text {side }}=$ 8192 , which corresponds to a pixel size of about 0.43 arcmin. The frequency responses are not provided in electronic format in the archive. We retrieved them from Fig. 10 of Chown et al. (2018) (long dashed lines in the three panels) using the WebPlotDigitizer $^{2}$. For additional details about the SPT-SZ public data, we refer the reader to the LAMBDA archive webpage given in footnote 1 and to Chown et al. (2018).

\subsection{Planck}

We used the public Planck maps of the High Frequency Instrument covering the six frequencies 100, 143, 217, 353, 545,

\footnotetext{
1 https://lambda.gsfc.nasa.gov/product/spt/spt_prod_ table.cfm

2 https://automeris.io/WebPlotDigitizer/
}

and $857 \mathrm{GHz}$. The maps are provided in Galactic coordinates at Healpix resolution $N_{\text {side }}=2048$, corresponding to a pixel size of about 1.72 arcmin. We upgraded the maps to $N_{\text {side }}=8192$ by zero padding in harmonic space, and we changed the coordinate system to equatorial to match the SPT-SZ public data. For the analysis, we assume the Planck beams are Gaussian with an FWHM of 9.659, 7.220, 4.900, 4.916, 4.675, and 4.216 arcmin at $100,143,217,353,545$, and $857 \mathrm{GHz}$, respectively, as in Planck Collaboration XXVII (2016a). The frequency responses are also based on the same reference. We converted the maps to $\mu \mathrm{K}$, that is to say the units of the SPT-SZ maps.

\section{Matched multi-filters}

We modified the MMFs, MMF3 (Melin et al. 2006, 2012), initially based on Herranz et al. (2002). The MMF3 algorithm was developed to extract clusters from the Planck maps for the three data releases (Planck Collaboration VIII 2011; Planck Collaboration XXIX 2014a; Planck Collaboration XXVII 2016a). It works on Planck data with $10 \times 10 \mathrm{deg}$ tangential maps. We wanted to keep the same Planck size for the tangential maps to ease the component separation on a large scale and the SPT-SZ pixel size to conserve the information at a small scale. We therefore divided the six Planck and three SPT-SZ frequency maps covering the SPT footprint into $10 \times 10 \mathrm{deg}$ tangential maps and kept the 0.43 arcmin pixels corresponding to $N_{\text {side }}=8192$. We filtered the resulting maps of $1400 \times 1400$ pixels with MMF3. These maps contain $4^{2}=16$ times more pixels than for the standard Planck analysis and nine frequency maps instead of six, leading to a computationally heavier analysis.

We write the nine tangential maps as $\boldsymbol{m}(\boldsymbol{x})$ and decomposed them as the cluster component $y_{\mathrm{o}} \boldsymbol{t}_{\boldsymbol{\theta}_{\mathrm{s}}}(\boldsymbol{x})$ and the noise $\boldsymbol{n}(\boldsymbol{x})$, corresponding to both instrumental noise and astrophysical components other than the cluster:

$\boldsymbol{m}(\boldsymbol{x})=y_{\mathrm{o}} \boldsymbol{t}_{\boldsymbol{\theta}_{\mathrm{s}}}(\boldsymbol{x})+\boldsymbol{n}(\boldsymbol{x})$,

where $y_{\mathrm{o}}$ is the central Compton- $y$ parameter, $\boldsymbol{t}_{\boldsymbol{\theta}_{\mathrm{s}}}(\boldsymbol{x})$ is the vector whose $i$ th component is $f_{v}\left(v_{i}\right)\left[b_{i} * T_{\theta_{\mathrm{s}}}\right](\boldsymbol{x})$, the tSZ template $T_{\theta_{\mathrm{s}}}$ convolved by $b_{i}$ (defined hereafter) and modulated by the tSZ frequency spectrum integrated over the frequency response, $f_{v}$, in $\mu \mathrm{K}$ units. The integration along the line-of-sight for $T_{\theta_{\mathrm{s}}}$ was performed out to $r=5 R_{500}$, and $\theta_{\mathrm{s}}$ is the scale radius, that is to say the characteristic scale of the cluster.

For Planck, $b_{i}$ is simply the Gaussian beam at frequency $v_{i}$, which is assumed to be azimuthally symmetric. For SPT-SZ, $b_{i}=B * T_{i}$, where the convolution of the azimuthally symmetric Gaussian beam is represented by $B(F W H M=1.75 \mathrm{arcmin})$ and the filter transfer function is $T_{i}$ at frequency $v_{i}$. The filter transfer function is not azimuthally symmetric so, in practice, we performed the convolution in all-sky Healpix maps at ten locations centered on the ten SPT clusters with the highest signal-to-noise ratio $(\mathrm{S} / \mathrm{N})$, cut tangential maps centered on $b_{i}$, and averaged them.

Assuming a pressure profile $T_{\theta_{\mathrm{s}}}$, MMF3 obtains the linear estimate of $y_{\mathrm{o}}$ with minimum variance:

$\hat{y}_{\mathrm{o}}=\int d^{2} x \boldsymbol{\Psi}_{\theta_{\mathrm{s}}}{ }^{t}(\boldsymbol{x}) \cdot \boldsymbol{m}(\boldsymbol{x})$,

where

$\boldsymbol{\Psi}_{\theta_{\mathrm{s}}}(\boldsymbol{k})=\sigma_{\theta_{\mathrm{s}}}^{2} \boldsymbol{P}^{-1}(\boldsymbol{k}) \cdot \boldsymbol{t}_{\theta_{\mathrm{s}}}(\boldsymbol{k})$,

with

$\sigma_{\theta_{\mathrm{s}}} \equiv\left[\int d^{2} k \boldsymbol{t}_{\boldsymbol{\theta}_{\mathrm{s}}}{ }^{t}(\boldsymbol{k}) \cdot \boldsymbol{P}^{-1} \cdot \boldsymbol{t}_{\boldsymbol{\theta}_{\mathrm{s}}}(\boldsymbol{k})\right]^{-1 / 2}$ 
and

$\boldsymbol{P}(\boldsymbol{k})=\left\langle\boldsymbol{n}^{*}(\boldsymbol{k}) \boldsymbol{n}(\boldsymbol{k})\right\rangle$.

We note that $\boldsymbol{k}$ is the two dimensional spatial frequency; and $\boldsymbol{P}(\boldsymbol{k})$ is the power spectrum matrix of the noise across frequency channels and was estimated directly from the data since the SZ signal is small compared to the other signals. The $\mathrm{S} / \mathrm{N}$ of the measurement is given by

$\frac{S}{N}=\frac{\hat{y}_{\mathrm{o}}}{\sigma_{\theta_{\mathrm{s}}}}$

The total integrated flux in the cylinder of radius $r=5 R_{500}$ can be estimated as

$Y_{5 R_{500}}=\hat{y}_{\mathrm{o}} \int_{x<5 \theta_{500}} \mathrm{~d} \Omega T_{\theta_{\mathrm{s}}}(x)$,

where $\theta_{500}$ is the angle subtended by $R_{500}$ at the cluster redshift. This total flux was then converted to the flux in the sphere of radius $r=R_{500}, Y_{500}$, by multiplying $Y_{5 R_{500}}$ by the ratio of $\frac{\int_{0}^{R_{500}} \mathrm{~d} r 4 \pi r^{2} t_{\theta_{\mathrm{S}}}(r)}{\int_{x<5 \theta_{500}} \mathrm{~d} \Omega T_{\theta_{\mathrm{S}}}(x)}$ (Appendix A of Melin et al. 2011). We note that $t_{\theta_{\mathrm{s}}}(r)$ is the three dimensional profile while $T_{\theta_{\mathrm{s}}}(x)$ is the two dimensional profile, that is, $t_{\theta_{\mathrm{s}}}(r)$ integrated along the line-ofsight. In the following, we adopt the profile of Arnaud et al. (2010) for $t_{\theta_{\mathrm{s}}}(r)$, except in Sect. 5.1 and Appendix B in which we adopt a $\beta$-profile to match the SPT-SZ cluster modeling.

We can run MMF3 in unblind or blind mode, that is, by fixing the position and size of the cluster or in letting the position and/or size free. In blind mode, we adopted the size and/or the position that maximize(s) the $\mathrm{S} / \mathrm{N}$ and refer to them(it) as a blind size (and blind position). The blind flux was estimated by fixing the filter size to the blind size and the position to the blind position if also left free. The blind mode is further described in Sect. 6.1.

\section{Consistency between public products and results published by the SPT and Planck collaborations}

We checked that the results obtained with SPT filter transfer functions applied to SPT-SZ maps are consistent with SPT published results. We extracted SPT point sources (Mocanu et al. 2013) in individual frequency maps using the filter transfer functions and we compared our recovered flux to the published flux. The results are presented in Appendix A. The filter functions are accurate for point source flux $S<50 \mathrm{mJy}$. For point sources with $S>50 \mathrm{mJy}$, the flux is biased a few percent high: This is expected because the regions surrounding bright point sources are excluded from the fitting of the time stream data (see Sect. 4.1.1 of Chown et al. 2018), so the filter transfer functions are not expected to model these regions of the maps.

We then extracted SPT clusters (Bleem et al. 2015a) using the SPT filter transfer functions and the SPT-SZ maps. The results are shown in Appendix B. SPT cluster flux was recovered without any biases for fluxes $Y^{0.75} \mathrm{SPT}<2 \times 10^{-4} \mathrm{arcmin}^{2}$, while for $Y^{0.75} \mathrm{SPT}>2 \times 10^{-4} \operatorname{arcmin}^{2}$ there is a few percent bias to larger fluxes ${ }^{3}$. This bias may come from the filter transfer function, which is biased high for bright sources, as shown above. The SPT cluster size was recovered without any biases, and SPT

\footnotetext{
$3 Y^{0.75} \mathrm{SPT}$ is the integrated SPT flux in a cylinder of radius
} 0.75 arcmin.
$\mathrm{S} / \mathrm{N}$ was recovered $10 \%$ low with respect to the results published by the SPT collaboration. The SPT public maps (Chown et al. 2018) are shallower than the maps used in Bleem et al. (2015a). We suspect that the $\mathrm{S} / \mathrm{N}$ bias to lower values is due to the difference in map depths. We would expect this bias to disappear if the MMFs were applied to the same SPT data as used in Bleem et al. (2015a).

Finally, we checked the consistency of the Planck cluster properties extracted from the Planck public data to the flux published by the Planck collaboration. The results are presented in Appendix C. We used one of the algorithms developed in the Planck collaboration but we do not expect to find a one-to-one relation between our recovered flux, size and $\mathrm{S} / \mathrm{N}$, and the quantities published by the Planck collaboration. This is because we upgraded the maps from $N_{\text {side }}=2048$ to 8192 , and we changed the coordinate system from Galactic to equatorial. In particular, the change of coordinates modifies the estimation of the noise power spectrum $\boldsymbol{P}(\boldsymbol{k})$, introducing some scatter in the recovered quantities with respect to the published values, but no significant bias.

We conclude that our extraction method provides results that are consistent with the results published by both the SPT and Planck collaboration. After these consistency tests between public products and the results published by the collaborations, we checked for consistency across the two data sets.

\section{Consistency between the SPT-SZ and Planck data sets}

We checked the consistency between the two data sets by extracting the SPT-SZ clusters in Planck data adopting the SPT cluster modeling and vice versa, that is, by extracting the Planck clusters in SPT-SZ data adopting the Planck cluster modeling.

\subsection{SPT-SZ cluster flux in Planck data}

We used the full $(\xi>4.5)$ SPT-SZ published cluster cata$\log$ (Bleem et al. 2015a). For the work described in this section, we adopted the $\beta$-profile for the cluster template with the same parametrization as in the SPT-SZ analysis. We applied MMF3 at the location of the clusters, fixing the size $\theta_{c}$ to the value published in the catalog. The estimated central Compton parameter $\hat{y}_{\mathrm{o}}$ was then converted to the integrated flux in a cylinder of radius $0.75 \operatorname{arcmin}, Y^{0.75}$. The flux can be directly compared to the flux given in the published catalog. The results are shown in Fig. 1.

There is a large dispersion between the individual measured fluxes and the published flux (black dots). This is expected because the Planck maps are noisier than the SPT-SZ maps. We averaged the Planck fluxes in SPT-SZ flux bins (red diamonds). The averaged bin flux is generally in good agreement with the SPT-SZ flux. The agreement is very good at large values $\left(Y^{0.75} \mathrm{SPT}>2 \times 10^{-4} \operatorname{arcmin}^{2}\right)$, and the average Planck flux starts to deviate to lower values with decreasing SPT-SZ flux $\left(Y^{0.75} \mathrm{SPT}<2 \times 10^{-4} \operatorname{arcmin}^{2}\right)$. We attribute this deviation to the Malmquist bias of the SPT-SZ flux due to the SPT detection threshold. Although Planck is less sensitive than SPT, it is not subject to the selection bias of the SPT sample. We conclude that the SPT cluster flux is consistent with the Planck data.

\subsection{Planck cluster flux in SPT-SZ data}

We used the published Planck catalog PSZ2 (Planck Collaboration XXVII 2016a). We restricted the sample 


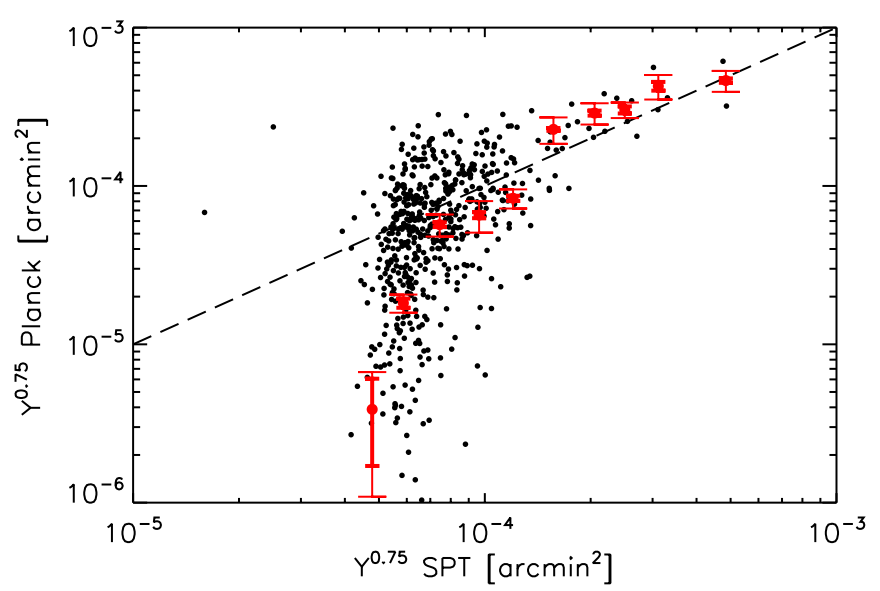

Fig. 1. Planck flux of the SPT-SZ clusters (Bleem et al. 2015a) as a function of the published SPT-SZ flux. The black dots are individual clusters. The red diamonds are weighted averages. The thick error bars show statistical errors and the thin error bars were obtained by the bootstrap method. Despite a large scatter, the agreement between the SPTSZ flux and the Planck flux is good. The drop-off at low flux is the Malmquist bias of the SPT-SZ flux due to the SPT detection threshold.

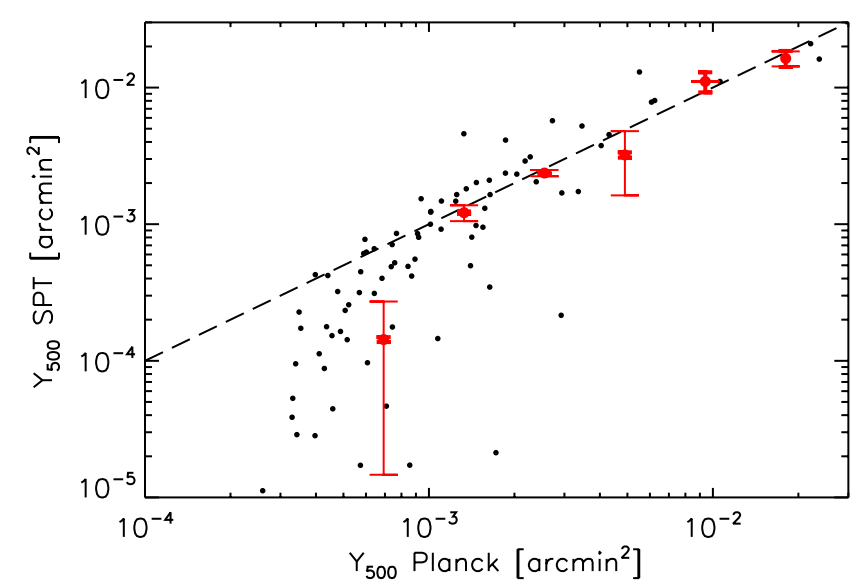

Fig. 2. SPT fluxes of Planck clusters (MMF3 sample of PSZ2, Planck Collaboration XXVII 2016a) as a function of published Planck flux. The agreement between the Planck flux and the SPT-SZ flux is good, as for the SPT sample shown in Fig. 1. The drop-off at low flux is the Malmquist bias of the Planck flux due to the Planck detection threshold. The legends are similar to Fig. 1.

to clusters detected by MMF3 $(S / N>4.5)$ and used the blind Planck flux and size, that is, the flux and size given at the maximum of the degeneracy contours provided by the Planck collaboration. We extracted the cluster flux from SPT-SZ maps fixing the position to the Planck blind position and the size to the Planck blind size. The results are given in Fig. 2. The figure shows a large scatter, but no significant bias for $Y_{500}$ Planck $>10^{-3} \operatorname{arcmin}^{2}$. At lower Planck fluxes $\left(Y_{500}\right.$ Planck $\left.<10^{-3} \operatorname{arcmin}^{2}\right)$, the SPT-SZ flux deviates toward smaller values. This deviation is likely due to the Malmquist bias in the Planck fluxes at the Planck detection threshold. We conclude that the Planck cluster flux is consistent with the SPT-SZ data.

With the two data sets being consistent, we then applied the MMF3 algorithm jointly to the Planck and SPT-SZ maps.

\section{The PSZSPT cluster catalog}

We first describe the construction of our candidate list using MMF3 in Sect. 6.1. We match our candidates to known clusters in Sect. 6.2. We check for missed SPT and PSZ2 clusters in Sect. 6.3. Finally, we estimate the completeness of our catalog in Sect. 6.4. The format and fields of the PSZSPT catalog are given in Appendix F.

\subsection{Construction of the catalog}

We divided the Planck and SPT-SZ Healpix maps into 52 overlapping tangential maps of $10 \times 10 \mathrm{deg}^{2}$ (pixel size $=0.43$ arcmin), covering the SPT-SZ footprint, and we ran the MMF3 algorithm blindly. The description of the blind MMF3 algorithm is given in Melin et al. (2012), Planck Collaboration VIII (2011), Planck Collaboration XXIX (2014a) and Planck Collaboration XXVII (2016a). We briefly recall here its main features and we give the differences with the implementation used for the Planck analyses.

The algorithm was run blindly on the individual maps. We fixed a detection threshold $q_{\text {thres }}$. We filtered each map with a set of 32 logarithmically spaced sizes $\theta_{\mathrm{s}}$, ranging from 0.8 to 30 arcmin. We looked for the maximum in the filtered maps corresponding to our first cluster candidate. We masked it and looked for the second maxima. We continued until there were no more remaining maxima above the detection threshold. In doing so, we built a blind catalog for each tangential map, which includes blind positions (corresponding to the position of the maxima), blind sizes (corresponding to the sizes maximizing the $\mathrm{S} / \mathrm{N}$ ), and blind fluxes (given by the filter output at the blind positions and for the blind sizes). We proceeded similarly for the 52 tangential maps. We then constructed a catalog from the 52 individual catalogs by merging detections with separation less than 2.5 arcmin. We then proceeded with a second pass of the algorithm. We divided the Planck and SPT-SZ Healpix maps in tangential maps centered on the first pass detections and ran the algorithm again. The second pass allowed us to obtain better estimates for the position, size, and flux, and to reject detections with refined $\mathrm{S} / \mathrm{N}$ lower than $q_{\text {thres }}$.

The Planck and SPT-SZ maps include bright point sources which must be masked to avoid spurious detections. We implemented the same methodology for the SPT-SZ and Planck tangential maps. We used a single frequency matched filter for each map and we detected point sources with $S / N>8$. The point source detection was performed in each of the three SPT-SZ maps and each of the six Planck maps. For the SPT-SZ data, we masked circular regions of 5 arcmin radius around each point source and rejected any SZ detection in a 7.5 arcmin radius. For comparison, Bleem et al. (2020) mask in 4 arcmin radius for $S / N>5$ point sources and rejected detections within 8 arcmin radius. For the Planck data, we masked in 10 arcmin radius and rejected detections in 15 arcmin radius because of the larger beams.

In summary, the differences between this implementation of MMF3 and the implementation used for the official Planck catalogs are as follows: the partial sky coverage (SPT-SZ footprint instead of all-sky); the pixel size of the maps $(0.43$ arcmin instead of 1.72 arcmin); the orientation of the tangential maps (equatorial pole to the north instead of the Galactic pole to the north); the filter transfer function for the SPT-SZ maps; the merging separation (2.5 arcmin instead of 10 arcmin); and the removal of bright point sources (made for SPT-SZ and Planck data on tangential maps instead of all-sky maps for Planck). 
We fixed the detection threshold $q_{\text {thres }}$ to 5 for our joint cata$\log$. We additionally applied the SPT-SZ boundary mask and the Planck cluster union mask (which keeps the $85 \%$ cleanest part of the sky). We thus obtain a catalog of 419 detections. In Sect. 6.2, we identify our detections with known clusters, and we present the completeness of our catalog in Sect. 6.4.

\subsection{Identification of known clusters}

We followed the methodology of Tarrío et al. (2019) to identify clusters in our joint catalog. We first identified clusters in the SPT-SZ catalog (Bleem et al. 2015a) (Sect. 6.2.1) and in the PSZ2 catalog (Planck Collaboration XXVII 2016a) (Sect. 6.2.2). We then cross matched the catalog with other relevant catalogs in the SPT-SZ footprint (Sect. 6.2.3): the Meta-Catalogue of Xray Clusters MCXC (Piffaretti et al. 2011), the Meta-Catalogue of SZ Clusters MCSZ ${ }^{4}$, the ComPRASS catalog (Tarrío et al. 2019), the Abell catalog (Abell et al. 1989), the cluster catalog from the Blanco Cosmology Survey (Desai et al. 2012; Bleem et al. 2015b), the MARD-Y3 catalog (Klein et al. 2019), and the WHY catalog (Wen et al. 2018). We finally used SIMBAD and NED (Sect. 6.2.4). We present the unidentified detections in Sect. 6.2.5.

\subsubsection{SPT-SZ catalog}

First, we matched each of the 419 blind detections to the closest cluster of the SPT-SZ cluster catalog. We then separated the detections into two sets: the detections matched to a SPT-SZ cluster with an estimated mass $M_{500}$ and redshift $z$, and the detections matched to a SPT-SZ detection without an estimated mass.

We plotted the first set in the $\theta / \theta_{500}$ versus $\theta$ plane where $\theta$ is the distance between the blind candidate and the closest SPTSZ cluster and $\theta_{500}$ is the angular radius corresponding to the published SPT-SZ mass $M_{500}$ and redshift $z$. The result is given in Fig. 3, which shows two clouds of detections: the detections which can be matched to an SPT-SZ cluster in the lower left corner and the detections which cannot be matched in the upper right corner. We define a detection as being matched to an SPTSZ cluster if $\theta<2$ arcmin, or $\theta / \theta_{500}<1$ and $\theta<10$ arcmin. We label it as rank $=1$. These detections correspond to the white region. We also define a detection as not being matched to a SPT-SZ cluster if $\theta>10$ arcmin, corresponding to the dark gray regions. We note it as rank $=0$. We then define the light gray region $\left(\theta / \theta_{500}>1\right.$ and $2 \operatorname{arcmin}<\theta<10$ arcmin) as the possibly matched clusters (rank $=2$ ).

For the second set without an estimated mass, we associated clusters (rank $=1$ ) if $\theta<2$ arcmin and we did not associate clusters (rank $=0$ ) if $\theta>10$ arcmin. The detections with 2 arcmin $<\theta<10$ arcmin were set as possibly associated (rank =2). The association is shown in Fig. 4. We note that there is no rank $=2$ detection of this category (light gray area) for the matching with the SPT catalog.

Finally, we checked for detections that were matched or possibly matched (rank $=1$ or rank $=2$ ) to the same SPT-SZ cluster, and we kept only the closest associated detection, giving priority to rank $=1$ over rank $=2$. We degraded the other multiple associations from rank $=1$ or rank $=2$ to rank $=3$. They are marked as crosses in Fig. 3. There are no rank $=3$ detections in Fig. 4. We investigate rank $=3$ detections further using SIMBAD and NED in Sect. 6.2.4.

\footnotetext{
4 https://www.galaxyclusterdb.eu/
}

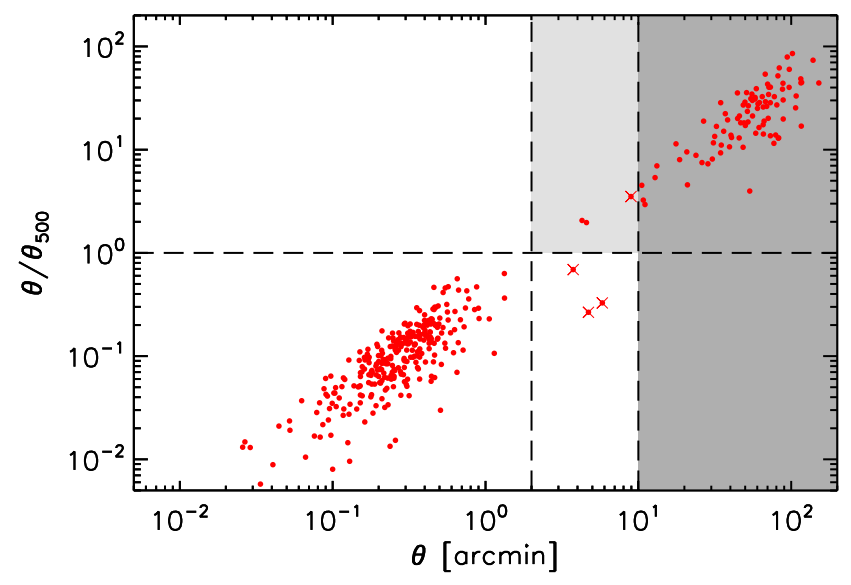

Fig. 3. Blind detections matching SPT-SZ clusters with published mass $M_{500}$ and redshift $z$. Candidates in the white (dark gray) area are matched (not matched) to SPT-SZ clusters. Candidates in the light gray area are possibly matched. The crosses mark multiple detections which are matched to SPT-SZ clusters already associated with a closer detection, and they are thus not considered as being matched to an SPT-SZ cluster.

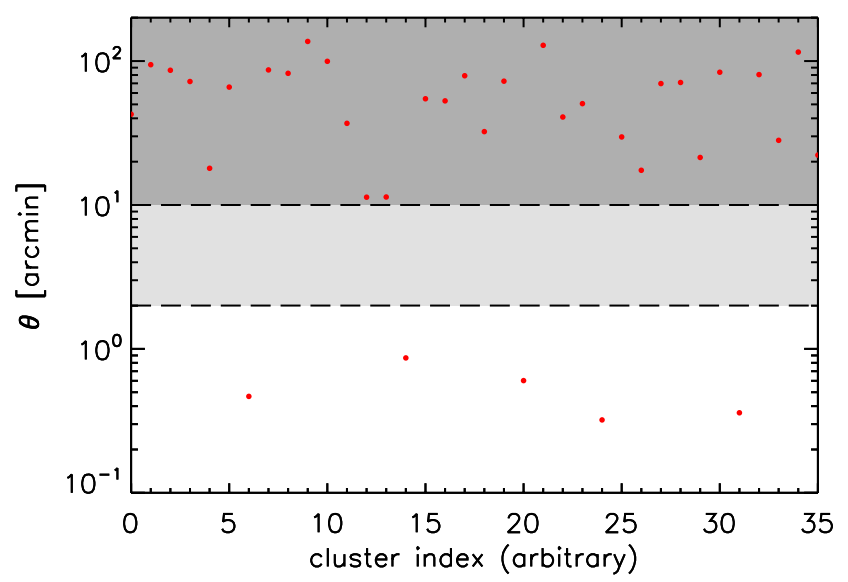

Fig. 4. Blind detections matching SPT-SZ clusters without published mass $M_{500}$. As for Fig. 3, candidates in the white (dark gray) area are matched (not matched) to SPT-SZ clusters.

As a final test, we estimated the mass for rank $=1$ associations using the cluster redshift $z$ and a X-ray prior on the $Y-M$ scaling relation (see Planck Collaboration XXIX 2014a; Planck Collaboration XXVII 2016a; Tarrío et al. 2019). We plotted it against the published SPT-SZ mass after recalibrating it by a factor of 0.8 to account for our mass definition, as was done in Tarrío et al. (2019) and as implemented in the MCSZ catalog. The result is shown in Fig. 5. The agreement between the two masses is good. We discuss this figure further, including outliers, in Appendix D.

Among the 419 joint detections, we found 290 matched to SPT-SZ clusters (rank =1) and two possibly matched to SPT-SZ clusters $($ rank $=2)$.

\subsubsection{PSZ2 catalog}

We applied the same methodology to the PSZ2 cluster catalog. The matching with PSZ2 clusters with mass $M_{500}$ and redshift $z$ is shown in Fig. 6, and the matching with clusters without masses is shown in Fig. 7. Given the larger beam size of Planck 


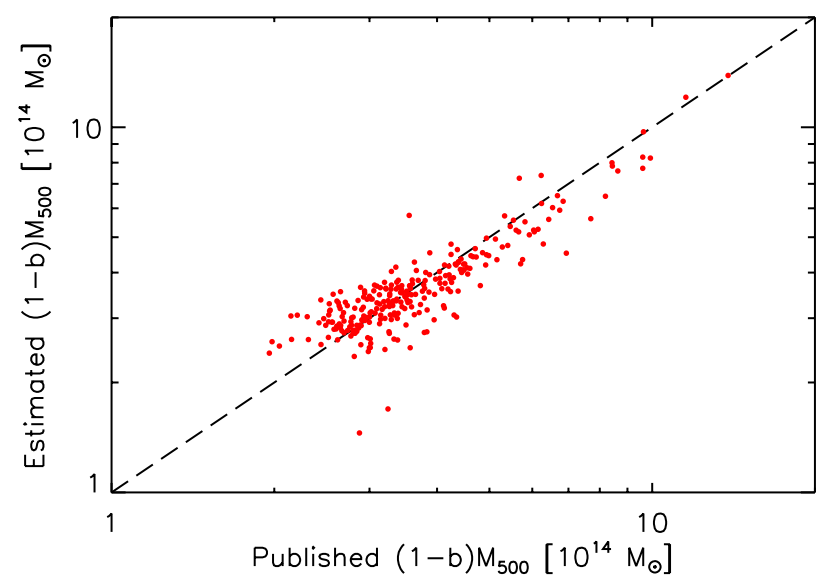

Fig. 5. Extracted masses versus published SPT-SZ masses for the joint detections matched to SPT-SZ clusters. Each point is a detection matched to an SPT-SZ cluster. We note that $(1-b)$ is the "mass bias factor" which relates the true mass $M_{500}$ to the XMM-Newton-like mass $M_{500, X}=(1-b) M_{500}$.

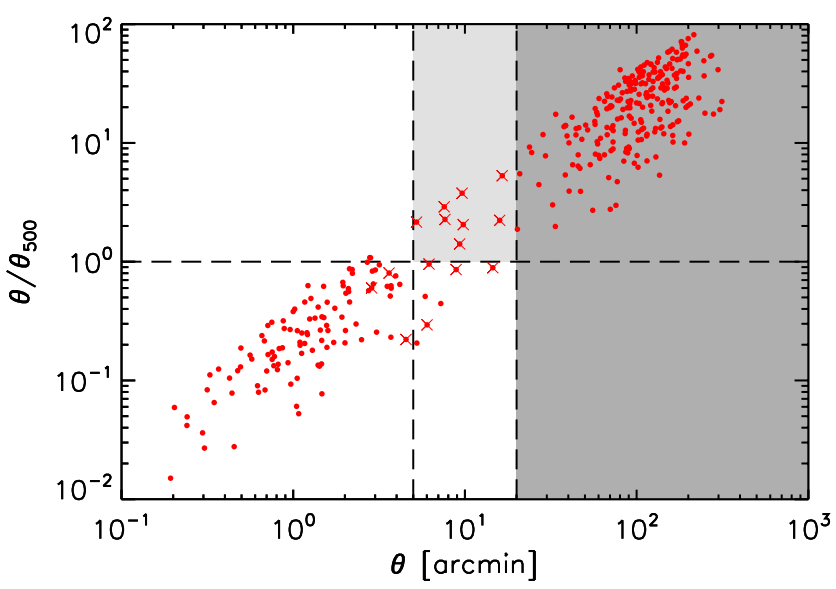

Fig. 6. Blind detections matching PSZ2 clusters with published mass $M_{500}$ and redshift $z$. The legend is the same as Fig. 3 .

we changed the limits for association. We define a detection as being matched to a PSZ2 cluster if $\theta<5$ arcmin, or $\theta / \theta_{500}<1$ and $\theta<20$ arcmin (white region in Fig. 6 , rank =1). A detection with $\theta>20$ arcmin (dark gray region, rank $=0$ ) is considered as not being matched, and the detection with $\theta / \theta_{500}>1$ and 5 arcmin $<\theta<20$ arcmin (light gray region, rank $=2$ ) is considered as being possibly matched.

The association with PSZ2 clusters without a published mass is shown in Fig. 7 with the same color coding of the regions for matched, not matched, and possibly matched detections. We also discarded double matching as we did for SPT-SZ (rank =3).

As for the SPT-SZ matching, we estimated the mass for rank $=1$ associations and plotted them against the published PSZ2 masses in Fig. 8. The agreement is also good. We discuss this figure further, including outliers and the systematic underestimation of the PSZ2 mass, in Appendix D.

Among the 419 joint detections, we found 112 matched to PSZ2 clusters (rank $=1$ ) and one possibly matched to PSZ2 clusters (rank =2). Finally, 82 detections are matched or possibly matched to a SPT-SZ and a PSZ2 cluster at the same time. At this stage, we thus have $419-292-113+82=96$ detections, which are not matched or possibly matched to a cluster from the SPT-SZ or the PSZ2 catalog.

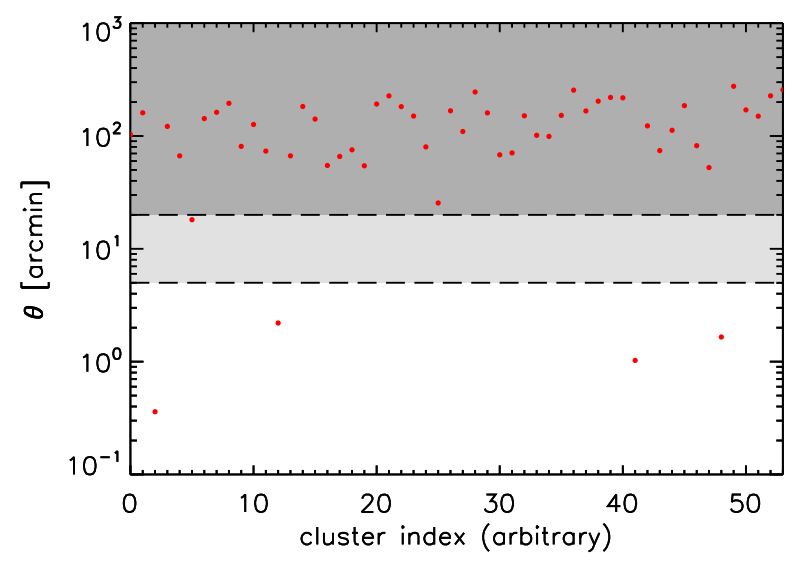

Fig. 7. Blind detections matching PSZ2 clusters without published mass $M_{500}$. As for Fig. 4, candidates in the white (dark gray) area are matched (not matched) to PSZ2 clusters. Candidates in the light gray region are possibly matched to a PSZ2 cluster.

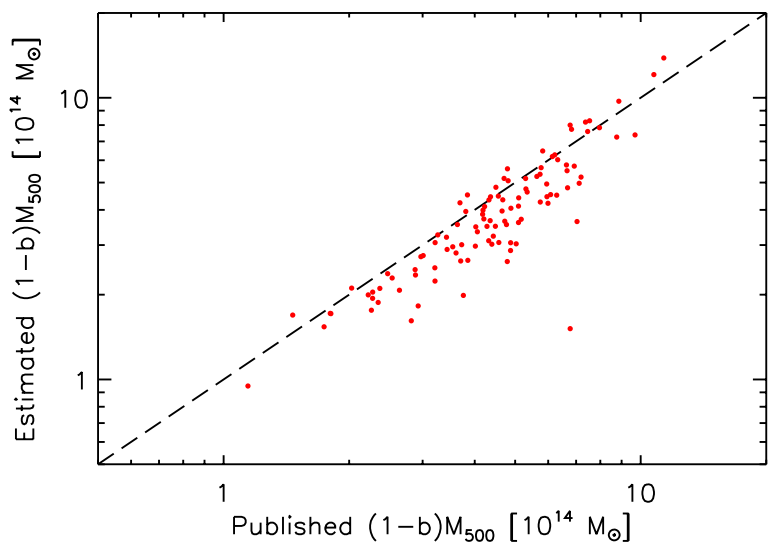

Fig. 8. Extracted masses versus published PSZ2 masses for the joint detections matched to PSZ2 clusters. Each point is a detection matched to a PSZ2 cluster. We note that $(1-b)$ is the same mass bias factor as in Fig. 5.

\subsubsection{Other catalogs}

After the matching with the SPT-SZ and the PSZ2 catalogs, we proceeded with the same methodology for the MCSZ Meta catalog, the MCXC Meta catalog (Piffaretti et al. 2011), the ComPRASS catalog (Tarrío et al. 2019), the Abell Southern catalog (Tables 4 and 5 of Abell et al. 1989), the Blanco Cosmology Survey (BCS, Desai et al. 2012; Bleem et al. 2015b), the MARDY3 catalog (Klein et al. 2019), and the WHY catalog (Wen et al. 2018). For the matching procedure, we adopted the same values as for the PSZ2 for all of these catalogs (5 and 20 arcmin), except for the SPT clusters in the MCSZ for consistency with Sect. 6.2.1, for which we adopted the SPT-SZ values (2 and 10 arcmin). For the MARD-Y3 catalog, we excluded multiple detections of the same source (by fixing fmult $=0$ ), and we set the contamination cut to $5 \%$ (by fixing fcontlfcr $<0.05$ ). For the WHY catalog, we used clusters with richness $R_{L^{*}} \geqslant 30$ and the mass-richness relation from Wen \& Han (2015).

Among the 96 detections, which are not matched or possibly matched to a SPT-SZ or a PSZ2 cluster, we find that:

- three are matched or possibly matched to MCSZ clusters

- PLCK G260.7-26.3 at $z=0.68$,

- PSZ1 G295.98-69.26, and

- PSZ1 G352.42-48.31; 
- three are matched or possibly matched to MCXC clusters

- MCXC J0330.0-5235 at $z=0.0624$,

- MCXC J0245.2-4627 at $z=0.0868$, and

- MCXC J2321.5-4153 at $z=0.0894$;

- ten are matched or possibly matched to ComPRASS clusters

- PSZRX G264.82-51.13 at $z=0.0624$ is also MCXC J0330.0-5235,

- PSZRX G348.32-66.45 at $z=0.0894$ is also MCXC J2321.5-4153,

- PSZRX G345.22-32.90 at $z=0.237$,

- PSZRX G271.53-56.58 at $z=0.3$,

- PSZRX G252.12-34.15,

- PSZRX G260.92-35.30,

- PSZRX G282.53-53.69,

- PSZRX G282.66-54.84,

- PSZRX G249.39-34.22, and

- PSZRX G352.49-33.18;

- 16 are matched or possibly matched to Abell clusters

- Abell S854 at $z=0.0561$,

- Abell 3128 at $z=0.0624$ is also PSZRX G264.82-51.13 and MCXC J0330.0-5235,

- Abell 3047 at $z=0.0868$ is also MCXC J0245.2-4627,

- Abell 3998 at $z=0.0894$ is also PSZRX G348.32-66.45 and MCXC J2321.5-4153,

- Abell 3279 at $z=0.1425$,

- Abell 3665 at $z=0.237$ is also PSZRX G345.22-32.90,

- Abell S295 at $z=0.3$ is also PSZRX G271.53-56.58,

- Abell S526 is also PSZRX G252.12-34.15,

- Abell 3886,

- Abell S47,

- Abell S132,

- Abell S184,

- Abell 3209,

- Abell 3236,

- Abell 3818, and

- Abell S1089;

- two are matched or possibly matched to BCS clusters

- BCS J233151-5736.2 at $z=0.27$ and

- BCS J051723-5325.5;

- 23 are matched or possibly matched to MARD-Y3 clusters

- MARD J201247.7-565058 at $z=0.0514$,

- MARD J032959.4-523546 at $z=0.0624$ is also Abell 3128, PSZRX G264.82-51.13, and MCXC J0330.05235 ,

- MARD J052449.4-613521 at $z=0.0788$,

- MARD J055700.0-424702 at $z=0.0825$,

- MARD J024524.4-462750 at $z=0.0868$ is also Abell 3047 and MCXC J0245.2-4627,

- MARD J232122.2-415047 at $z=0.0894$ is also Abell 3998, PSZRX G348.32-66.45, and MCXC J2321.54153,

- MARD J211813.3-474018 at $z=0.1103$,

- MARD J203848.2-463207 at $z=0.1300$,

- MARD J043805.0-455901 at $z=0.1425$ is also Abell 3279 ,

- MARD J200938.7-531030 at $z=0.237$ is also Abell 3665 and PSZRX G345.22-32.90,

- MARD J021020.5-464352 at $z=0.292$,

- MARD J024528.5-530203 at $z=0.3$ is also Abell S295 and PSZRX G271.53-56.58,

- MARD J044052.4-474315 at $z=0.3075$,

- MARD J011511.6-595828 at $z=0.3972$,

- MARD J223243.0-542921 at $z=0.4248$,

- MARD J215445.0-593625 at $z=0.4317$,
- MARD J043431.7-572717 at $z=0.4709$,

- MARD J061633.0-522732 at $z=0.68$ is also PLCK G260.7-26.3,

- MARD J052243.5-461106,

- MARD J014307.6-582601,

- MARD J022718.3-592935,

- MARD J022133.6-583730, and

- MARD J040244.4-533049; and

- 31 are matched or possibly matched to WHY clusters

- J201358.6-570223 at $z=0.0514$ is also MARD J201247.7-565058,

- J201050.6-564025 at $z=0.0561$ is also Abell S854,

- J032950.6-523447 at $z=0.0624$ is also MARD J032959.4-523546, Abell 3128, PSZRX G264.8251.13, and MCXC J0330.0-5235,

- WHY J052352.8-614054 at $z=0.0788$ is also MARDJ052449.4-613521,

- J024513.3-462719 at $z=0.0868$ is also MARD J024524.4-462750, Abell 3047, and MCXC J0245.24627 ,

- J042953.7-463447 at $z=0.1389$

- J043815.2-455712 at $z=0.1425$ is also MARD J043805.0-455901 and Abell 3279,

- J223249.9-545158 at $z=0.151$,

- WHY J201309.0-463059 at $z=0.1746$,

- WHY J215446.7-461610 at $z=0.2161$,

- WHY J214150.7-481507 at $z=0.2319$,

- J200950.6-530948 at $z=0.237$ is also MARD J200938.7-531030, Abell 3665, and PSZRX G345.2232.90 ,

- WHY J060809.4-615348 at $z=0.2424$,

- WHY J010643.2-575952 at $z=0.2568$,

- WHY J064816.1-611730 at $z=0.2707$,

- WHY J220455.6-622321 at $z=0.2761$,

- WHY J061026.5-461946 at $z=0.2782$,

- J000314.6-525516 at $z=0.2799$,

- WHY J021011.3-464254 at $z=0.292$ is also MARD J021020.5-464352,

- WHY J044100.6-474229 at $z=0.3075$ is also MARD J044052.4-474315,

- WHY J234242.1-465853 at $z=0.3322$,

- WHY J212830.6-463750 at $z=0.3381$,

- J041355.6-461148,

- J002651.3-514159,

- J052316.1-460241,

- J230911.4-474414,

- J040244.8-533313,

- WHY J052112.2-435700,

- WHY J014308.1-582621,

- WHY J022131.4-583722, and

- WHY J223303.5-542946.

These matched detections are mainly at low $(z<0.1)$ and intermediate $(z \sim 0.3)$ redshift, or have no published redshift. Some of them are in common between the catalogs. After this new set of associations, 48 detections among the 96 remain unmatched. Among these 48 detections, two are rank $=3$ detections (for which we broke a rank $=1$ and rank $=2$ association) and the 46 others are not classified.

\subsubsection{SIMBAD and NED}

We searched for counterparts in SIMBAD and NED for the remaining 48 detections, which are not matched to any of the 
clusters in the studied catalogs. We set the search radius to 20 arcmin for the two databases and we looked for galaxy cluster-type objects. We did not find a cluster in the search radius for 34 detections. We set $r a n k=0$ (meaning unidentified) for these 34 detections.

For the other $48-34=14$ detections, we found two obvious bright and large clusters (ACO S 1063 and ACO 3911) close to the two rank $=3$ detections, confirming that these two detections are multiple (therefore false) detections produced by the algorithm. We also found found SPT clusters that are not included in the cluster catalog provided by Bleem et al. (2015a): Three were excluded from the official SPT catalog because they are close to a bright point source (Table 3 of Bleem et al 2015a), one is in Saro et al. (2015), but not in Bleem et al. (2015a). We set rank $=1$ for these four detections. The remaining $14-2-4=8$ detections are not obviously matched to the clusters found in the search radius because the counterparts are at a distance greater than 7 arcmin. We thus set rank $=0$ (unidentified) for these eight detections. We additionally checked for detections in the PSZSPT catalog separated by less than 5 arcmin and noticed that PSZSPT J2012-5649 and PSZSPT J2012-5652 are associated with clusters located at the same redshift. After a SIMBAD and NED search at the location of the two clusters, we noticed that PSZSPT J2012-5652 might actually be a substructure of PSZSPT J2012-5649 (ACO 3667). We thus set PSZSPT J2012-5652 as a rank $=3$ (multiple) detection.

In summary, after the SIMBAD and NED search, there were seven additional identifications (three multiple rank $=3$ clusters, four SPT rank $=1$ clusters not in Bleem et al. 2015a) and 34+8 = 42 unidentified (rank $=0$ detections).

\subsubsection{Unidentified detections}

We looked for the spatial distribution of the 42 unidentified detections in the SPT footprint. We found no specific pattern which could indicate a problem with the extraction algorithm related to the possible systematics in the maps, except in a specific location on the edge of the SPT footprint. This location is displayed in Fig. 9. The figure shows the local variance of the instrumental SPT-SZ noise at $150 \mathrm{GHz}$ as the half map difference $\Delta H M$ squared filtered by a 10 arcmin FWHM Gaussian beam $G$ minus the square of the filtered half map difference:

$$
\int \mathrm{d} \boldsymbol{x}^{\prime} G\left(\boldsymbol{x}-\boldsymbol{x}^{\prime}\right) \Delta H M^{2}\left(\boldsymbol{x}^{\prime}\right)-\left(\int \mathrm{d} \boldsymbol{x}^{\prime} G\left(\boldsymbol{x}-\boldsymbol{x}^{\prime}\right) \Delta H M\left(\boldsymbol{x}^{\prime}\right)\right)^{2} .
$$

The black triangle on the right of the image is outside the SPT footprint. There is a clear separation between the top and bottom of the image with the upper part of the image being less noisy than the bottom part (factor 2.5 between the variances of the two parts). This noise difference is due to different integration times or instrumental sensitivities around this location. The detections are displayed as disks. The black and white circles are identified and unidentified detections, respectively. A cluster of unidentified detections is visible in the bottom part. The MMF algorithm estimates the noise on the full map. It is thus possible that the noise is not correctly estimated in this specific patch due to the inhomogeneity of the SPT-SZ instrumental noise. However, four detections have been identified with known clusters in this patch, two of them being in the top part and the other two in the bottom part. We thus do not flag unidentified detections in this patch.

In the first line of Fig. 10, we summarize the number of PSZSPT detections according to their rank. In the other lines, we provide the number of rank $=1$ and rank $=2$ detections associated to external catalogs that we considered in this article. We

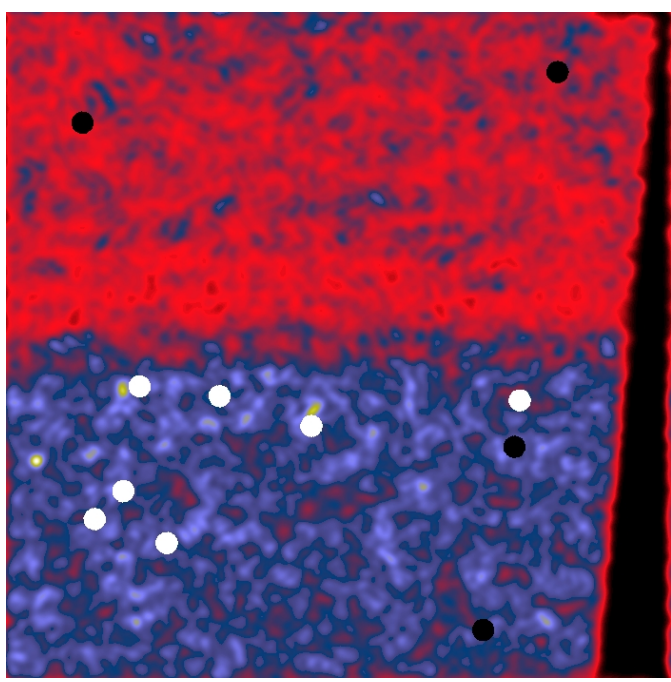

Fig. 9. Identified (black disks) and unidentified (white disks) detections in the $10 \times 10 \mathrm{deg}^{2}$ tangential map centered on RA $=307.364 \mathrm{deg}$ and Dec $=-45.6752 \mathrm{deg}$. The background image displays the local variance of the instrumental noise at the SPT-SZ $150 \mathrm{GHz}$ frequency, which is higher in the bottom part of the map.

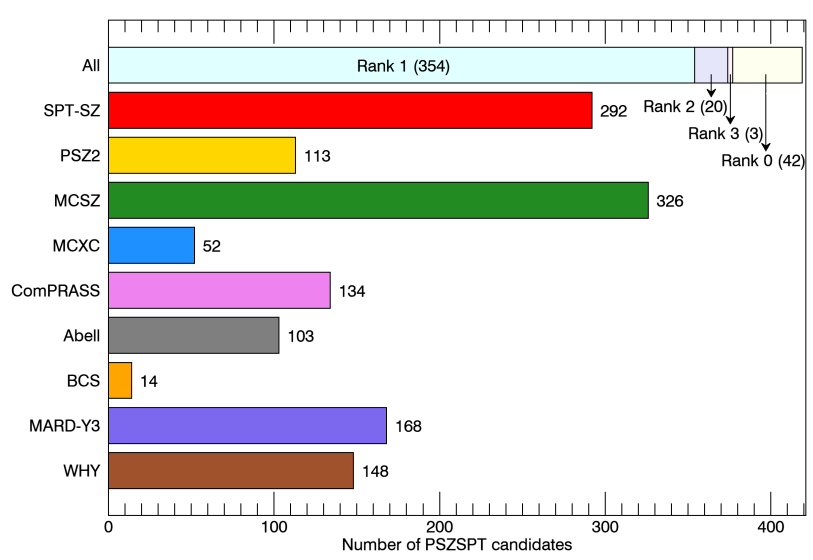

Fig. 10. First line: number of PSZSPT candidates according to their rank. We note that $0=$ unidentified, $1=$ associated to known cluster, $2=$ possibly associated to known cluster, and $3=$ multiple detection. Other lines: number of rank $=1$ and rank $=2$ detections associated with external catalogs.

note that the total number exceeds $354+20=374$ (numbers given in the first line) because the external catalogs share some common objects.

\subsection{Missed SPT and PSZ2 clusters}

We now investigate the SPT and PSZ2 clusters in the SPTSZ footprint with a signal-to-noise greater than five which are not in the PSZSPT catalog. The official SPT catalog contains $677 / 409$ detections with a signal-to-noise of $\xi>4.5 / 5$. After the Chown et al. (2018) mask was applied, 379 detections with $\xi>5$ remained. We additionally applied the Planck union mask which leaves $376 \xi>5$ detections in the SPT catalog. We found 292 matches in Sect. 6.2 between the full SPT catalog $(\xi>4.5)$ and the PSZSPT catalog. This reduces to 268 matches with SPT detections at $\xi>5$ in the Chown et al. (2018) and Planck union masks. Thus 376-268 $=108$ SPT detections at $\xi>5$ are unmatched with the PSZSPT detections. Seven of them are in the 
point source mask built by our extraction algorithm (Sect. 6.1). Among the remaining 101, four have $\xi>7$ and 82 have assigned redshift and mass. We plotted the SPT detections having a redshift and mass in Fig. 11 as small blue dots. We overplotted the 82 unmatched detections with large black crosses. We added large black circles to the four high $\xi>7$ clusters (they all have a redshift).

We applied the same methodology to the PSZ2 detections. We note that 107 PSZ2 detections with $S / N>5$ are in the Chown et al. (2018) and PSZ2 union masks. Among the 113 matches found in Sect. 6.2, 91 have $S / N>5$. This leaves $107-$ $91=16$ PSZ2 detections unmatched. Five of them are in the point source mask built by our extraction algorithm (Sect. 6.1). Among the remaining 11, one have $S / N>7$ and six have an assigned redshift and mass. We plotted the PSZ2 clusters with a redshift and mass as large red dots in Fig. 11. We overplotted the six unmatched detections with large black crosses and the cluster with $S / N>7$ with a large black circle. We note that we applied a 0.8 recalibrating factor to the SPT mass, as in Fig. 5. We provide the names of the missed SPT and PSZ2 clusters in Appendix E.

When combining SPT-SZ and Planck, the S/N of the detections is expected to be, on average, greater than the $\mathrm{S} / \mathrm{N}$ on the individual experiments. However, due to estimation errors, there is a scatter around the expected value, which makes some of the clusters, especially those close to the limit, to down-scatter below the PSZSPT detection threshold. Additionally, we show in Appendix B that our extracted S/N on SPT-SZ maps is, on average, $10 \%$ lower than the $\mathrm{S} / \mathrm{N} \xi$ published by the SPT collaboration. For these reasons, we expected to miss several SPT-SZ clusters close to $\xi=5$, and also a small number of higher signalto-noise clusters.

The majority of the unmatched candidates (indicated by the black crosses) are indeed at the detection limit of the SPT and Planck catalogs. Thus they may have been missed because of noise fluctuations in the filtered maps. There are, however, four SPT detections and one Planck detection (large black circles) with a signal-to-noise greater than seven. Three of the SPT clusters have $S / N<5$ in the filtered maps after the first pass and are thus not detected by the joint algorithm. The last SPT cluster has $S / N \sim 8.1$ in the filtered maps after the first pass. It is thus detected in the first pass of the algorithm, but it was not included in the catalog after the second pass because it is located close to the edge of the SPT-SZ footprint and the noise power spectrum was not estimated properly after the cluster recentering so the detection is rejected. The PSZ2 undetected cluster has $S / N \sim 5.5$ after the first pass of the algorithm, but it does not pass the threshold after the re-centering of the second pass $(S / N \sim 4.6)$ and is thus not included in the catalog.

\subsection{Completeness}

In this section, we adopt the Planck Collaboration VI (2020) CMB cosmology (TT,TE,EE+lowE+lensing model ${ }^{5}$ ).

We followed the method from Sect. 3.2 of Planck Collaboration XX (2014b) (also described in Sect. 3.2 of Planck Collaboration XXIX 2014a, Sect. 3.3 of Planck Collaboration XXIV 2016b, and Sect. 4.2 of Planck Collaboration XXVII 2016a) to predict the completeness of the joint and individual surveys semi-analytically. We assumed that the noise of the maps is Gaussian after filtering with the MMFs. Thus, the completeness can be expressed

Flat model with $h=0.6736, \Omega_{\mathrm{m}}=0.3153$, and $\sigma_{8}=0.8111$.

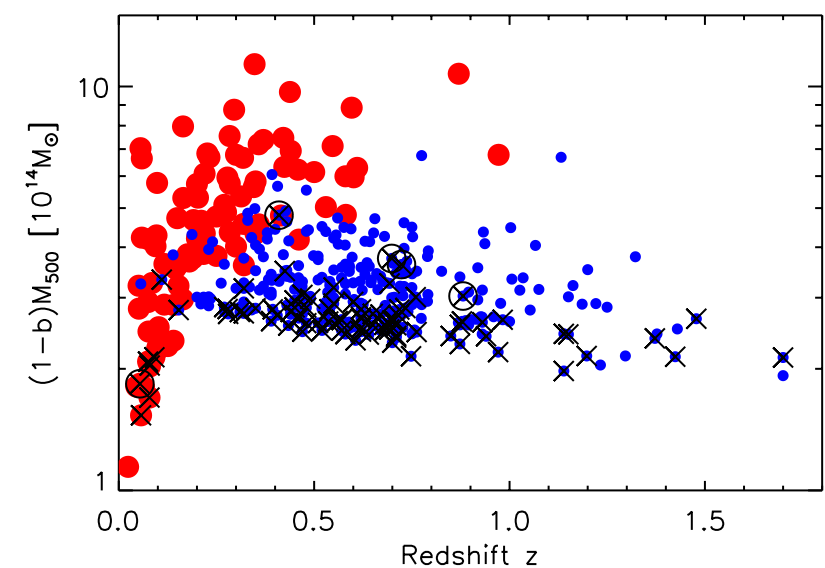

Fig. 11. Mass-resdhift distribution of the SPT (small blue dots) and PSZ2 (large red dots) clusters. The SPT and Planck clusters that went undetected in the PSZSPT are marked with large black crosses. The large black circles additionally indicate the signal-to-noise greater than seven clusters missed by the PSZSPT. We note that $(1-b)$ is the mass bias factor as in Figs. 5 and 8.

as an erf function of the cluster size $\theta_{500}$, the cluster flux $Y_{500}$, the detection threshold $q$ (set to 5 in this work), and the position on the sky (RA, Dec). We express the completeness as a function of redshift $z$ and mass $M_{500}$, adopting the $Y_{500}-M_{500}$ and $\theta_{500}-M_{500}$ relations from Eqs. (7) and (9) in Planck Collaboration XX (2014b). We then integrated the result over the sky coverage of SPT-SZ.

The resulting completeness is shown in the left panel of Fig. 12 for the three catalogs: Planck+SPT-SZ, Planck, and SPTSZ. The completeness of the joint catalog Planck+SPT-SZ is driven by SPT-SZ at redshift $z>0.5$ and by Planck at $z<0.1$. This is expected because SPT-SZ has less instrumental noise and a better resolution than Planck, leading to better efficiency at detecting high- $z$ clusters. On the other hand, the SPT filtering strategy smoothes the large angular scales, which prevents the detection of very low- $z$ clusters. Planck, as a satellite, is not affected by this effect and can detect the low redshift clusters. Both surveys contribute to the intermediate redshift range $0.1<z<0.5$.

We expect the unidentified detections to mainly populate this specific range. We note that $(1-b)$ is the "mass bias factor", which relates the true mass $M_{500}$ to the XMM-Newton-like mass $M_{500, X}=(1-b) M_{500}$. The scaling laws from Eqs. (7) and (9) in Planck Collaboration XX (2014b) are based on XMM-Newton masses, and we decided to leave this parameter free in this left panel to aid comparison with other works.

From the completeness, we can predict the expected cluster counts from a given mass function. We chose the Tinker et al. (2008) mass function, and we fixed $(1-b)=0.63$ to adjust the Planck MMF3 cosmological cluster counts to the Planck Collaboration VI (2020) CMB cosmology. This value of $(1-b)$ is very close to the value $(1-b)=0.62 \pm 0.03$ obtained by Planck Collaboration VI (2020) (see their Eq. 34) using the same dataset, and to the value $(1-b)=0.622 \pm 0.033$ found by Salvati et al. (2019) (see their Table 1) on an extended dataset. The predicted redshift distribution is given in the right panel of Fig. 12 for the SPT-SZ footprint.

As expected from the completeness, the overall Planck+SPT-SZ cluster count (thick red line) is dominated by the SPT-SZ dataset, except at a very low redshift where Planck provides the information. The predicted total number 

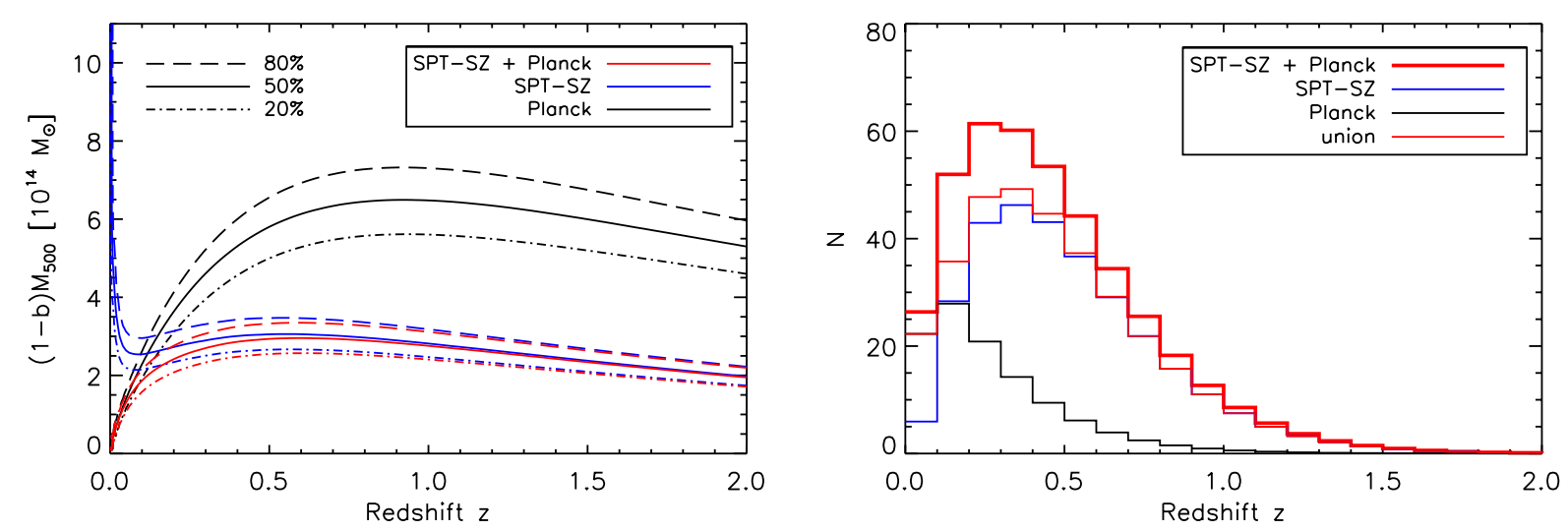

Fig. 12. Left: completeness of the joint Planck+SPT-SZ survey (red) compared to the completeness of individual Planck (black) and SPT-SZ (blue) surveys. The dashed, solid, and dotted-dashed line correspond to the 80,50, and 20\% completeness level, respectively. Right: predicted cluster counts in each redshift bin for a Planck Collaboration VI (2020) primary CMB cosmology in the SPT-SZ footprint. The Planck+SPT-SZ curve corresponds to the joint extraction, while the union curve corresponds to the union of the individual Planck and SPT-SZ catalogs. The mass bias factor $(1-b)$ was set to 0.63 in the right figure.

of clusters is 413,302 , and 111 for Planck+SPT-SZ, SPT-SZ, and Planck, respectively, which is in very good agreement with the number of detections given in Sect. 6.1. The union catalog of the individual Planck and SPT-SZ catalogs is shown as the thin red line for comparison with the joint catalog. We provide these predicted cluster numbers as a consistency check between the cluster counts of our joint catalog and the Planck Collaboration VI (2020) CMB cosmology. Constraining cosmological parameters from this joint catalog is beyond the scope of this work.

\section{Summary, discussion, and future work}

We performed, for the first time, an SZ cluster extraction using space-based (Planck) and ground-based (SPT) data jointly. We also provide the associated PSZSPT catalog of 419 sources at $S / N>5$.

For this purpose, we modified the MMF3 algorithm that was initially developed for Planck data to make it compatible with ground based data. The main difficulties were including the transfer function of the SPT survey as well as handling the high resolution ground-based and low resolution space data at the same time, which required the use of small $(0.43 \mathrm{arcmin})$ pixels on large $\left(10 \times 10 \mathrm{deg}^{2}\right)$ tangential maps. In the process of building the joint catalog, we thoroughly characterized the SPT public maps (transfer function, point source, and cluster photometry) with respect to the SPT official catalogs (Sect. 4, Appendices A and B). We found good agreement between quantities extracted with our tools and the official SPT catalogs. However, the S/N of our SPT extractions is $10 \%$ lower than the published values. We attribute this to the difference in map depths between the SPT public maps (Chown et al. 2018) and the maps used in Bleem et al. (2015a). Our extraction method would thus be more efficient if it could be applied to the maps used in Bleem et al. (2015a). We also checked that our new extraction method provides results consistent with the Planck publications (Sect. 4, Appendix C). We then showed that the Planck and SPT data provide consistent flux measurements for SPT and Planck clusters, respectively (Sect. 5).

We cross-matched the PSZSPT catalog with other cluster catalogs in the SPT-SZ footprint. We checked for remaining unidentified detections in SIMBAD and NED. We note that 292(113) detections are matched or possibly matched to
SPT-SZ(PSZ2) detections, respectively, with 82 being common to both of the catalogs. We identified 47 detections with clusters in catalogs other than SPT-SZ and PSZ2, and seven additional detections using SIMBAD and NED. Finally, we could not identify counterparts for the remaining 42 detections which need to be validated by future external follow-ups (Sect. 6.2).

We finally estimated the completeness of the PSZSPT catalog and checked that the extracted counts are consistent with the standard $\Lambda \mathrm{CDM}$ model when adopting the Planck cluster modeling and scaling laws (Sect. 6.4). The PSZSPT catalog is described in Appendix F and a complete version is available in electronic format at the CDS.

The increase in the number counts from the joint catalog with respect to the union of the space- and ground-based catalog is moderate, as shown in Fig. 12 (difference between the red thin and thick lines). The majority of new detections are expected to be in the redshift range $[0.1,0.6]$ around the location where the Planck and SPT-SZ completenesses cross. The most interesting application of the space- and ground-based joint cluster analyses with current data sets may thus be astrophysical studies and, in particular, cluster profiles, the space-based (ground-based) data providing the large-scale (small-scale) information, respectively. We leave this work for a future article.

This first proof of concept of joint cluster extraction with space- and ground-based data opens the path to other possible catalogs when new data will be publicly available, for example Planck and ACT (Naess et al. 2020) or Planck and SPTECS (Bleem et al. 2020). But the approach would be most useful in the case of the longer term ground-based Simons Observatory (Ade et al. 2019) or CMB-S4 (Abazajian et al. 2019) and proposed space mission such as PICO (Hanany, et al. 2019) or Backlight (Basu et al. 2019; Delabrouille et al. 2019), which have resolutions matching between space and ground (1 arcmin FWHM at $300 \mathrm{GHz}$ ) and together cover a large frequency range from a few tens of gigahertz to terahertz. The space and ground approach will be crucial to disentangle the various emissions in clusters: the different SZ effects as well as the radio and infrared emission of galaxies hosted by clusters.

Acknowledgements. We would like to thank the anonymous referee for useful comments which helped to clarify this work. We acknowledge the use of the Legacy Archive for Microwave Background Data Analysis (LAMBDA), part of the High Energy Astrophysics Science Archive Center (HEASARC). HEASARC/LAMBDA is a service of the Astrophysics Science Division at 
the NASA Goddard Space Flight Center. We also used the HEALPix software (Górski et al. 2005) available at https://healpix.sourceforge.io and the WebPlotDigitizer by Ankit Rohatgi. This research has also made use of the M2C Galaxy Cluster Database, constructed as part of the ERC project M2C (The Most Massive Clusters across cosmic time, ERC-Adv grant No. 340519), the SIMBAD database operated at CDS, Strasbourg, France (Wenger et al. 2000), and the NASA/IPAC Extragalactic Database (NED), which is funded by the National Aeronautics and Space Administration and operated by the California Institute of Technology. J.-B. Melin thanks Monique Arnaud for constructive discussions which helped to improve this article and for suggesting the acronym PSZSPT. He also warmly thanks the high school students Julie Hebert, Aubin Mayer, Hugo Levandowski and Anaëlle Meurant who helped him search the PSZSPT locations in the SIMBAD and NED databases. A portion of the research described in this paper was carried out at the Jet Propulsion Laboratory, California Institute of Technology, under a contract with the National Aeronautics and Space Administration.

\section{References}

Abazajian, K., Addison, G., Adshead, P., et al. 2019, ArXiv e-prints [arXiv:1907.04473]

Abell, G. O., Corwin, H. G. Jr, \& Olowin, R. P. 1989, ApJS, 70, 1

Ade, P., Aguirre, J., Ahmed, Z., et al. 2019, JCAP, 2019, 056

Aghanim, N., Douspis, M., Hurier, G., et al. 2019, A\&A, 632, A47

Allen, S. W., Evrard, A. E., \& Mantz, A. B. 2011, ARA\&A, 49, 409

Arnaud, M., Pratt, G. W., Piffaretti, R., et al. 2010, A\&A, 517, A92

Basu, K., Remazeilles, M., Melin, J. B., et al. 2019, ArXiv e-prints [arXiv:1909.01592]

Bleem, L. E., Stalder, B., Brodwin, M., et al. 2015a, ApJS, 216, 20

Bleem, L. E., Stalder, B., de Haan, T., et al. 2015b, ApJS, 216, 27

Bleem, L. E., Bocquet, S., Stalder, B., et al. 2020, ApJS, 247, 25

Böhringer, H., Voges, W., Huchra, J. P., et al. 2000, ApJS, 129, 435

Böhringer, H., Schuecker, P., Guzzo, L., et al. 2001, A\&A, 369, 826

Burenin, R. A. 2017, Astron. Lett., 43, 507

Chown, R., Omori, Y., Aylor, K., et al. 2018, ApJS, 239, 10

Delabrouille, J., Abitbol, M. H., Aghanim, N., et al. 2019, ArXiv e-prints [arXiv:1909.01591]

Desai, S., Armstrong, R., Mohr, J. J., et al. 2012, ApJ, 757, 83
Finoguenov, A., Rykoff, E., Clerc, N., et al. 2020, A\&A, 638, A114 Górski, K. M., Hivon, E., Banday, A. J., et al. 2005, ApJ, 622, 759 Hanany, S., Alvarez, M., Artis, E., et al. 2019, BAAS, 51, 194 Herranz, D., Sanz, J. L., Hobson, M. P., et al. 2002, MNRAS, 336, 1057 Klein, M., Grandis, S., Mohr, J. J., et al. 2019, MNRAS, 488, 739 Madhavacheril, M. S., Hill, J. C., Næss, S., et al. 2020, Phys. Rev. D, 102, 023534 Maturi, M. 2007, Astron. Nachr., 328, 690

Melin, J. B., Bartlett, J. G., \& Delabrouille, J. 2006, A\&A, 459, 341

Melin, J. B., Bartlett, J. G., Delabrouille, J., et al. 2011, A\&A, 525, A139

Melin, J.-B., Aghanim, N., Bartelmann, M., et al. 2012, A\&A, 548, A51

Melin, J. B., Bartlett, J. G., Cai, Z. Y., et al. 2018, A\&A, 617, A75

Menanteau, F., González, J., Juin, J.-B., et al. 2010, ApJ, 723, 1523

Mocanu, L. M., Crawford, T. M., Vieira, J. D., et al. 2013, ApJ, 779, 61

Mocanu, L. M., Crawford, T. M., Aylor, K., et al. 2019, JCAP, 2019, 038

Naess, S., Aiola, S., Austermann, J. E., et al. 2020, JCAP, 12, 046

Piffaretti, R., Arnaud, M., Pratt, G. W., Pointecouteau, E., \& Melin, J. B. 2011, A\&A, 534, A109

Planck Collaboration VIII., 2011, A\&A, 536, A8

Planck Collaboration XXIX., 2014a, A\&A, 571, A29

Planck Collaboration XX., 2014b, A\&A, 571, A20

Planck Collaboration XXVII., 2016a, A\&A, 594, A27

Planck Collaboration XXIV., 2016b, A\&A, 594, A24

Planck Collaboration VI., 2020, A\&A, 641, A6

Salvati, L., Douspis, M., Ritz, A., Aghanim, N., \& Babul, A. 2019, A\&A, 626, A27

Saro, A., Bocquet, S., Rozo, E., et al. 2015, MNRAS, 454, 2305

Staniszewski, Z., Ade, P. A. R., Aird, K. A., et al. 2009, ApJ, 701, 32

Story, K. T., Reichardt, C. L., Hou, Z., et al. 2013, ApJ, 779, 86

Sunyaev, R. A., \& Zeldovich, Y. B. 1970, Comm. Astrophys. Space Phys., 2, 66

Sunyaev, R. A., \& Zeldovich, Y. B. 1972, Comm. Astrophys. Space Phys., 4, 173

Tarrío, P., Melin, J. B., Arnaud, M., \& Pratt, G. W. 2016, A\&A, 591, A39

Tarrío, P., Melin, J. B., \& Arnaud, M. 2018, A\&A, 614, A82

Tarrío, P., Melin, J. B., \& Arnaud, M. 2019, A\&A, 626, A7

Tinker, J., Kravtsov, A. V., Klypin, A., et al. 2008, ApJ, 688, 709

Voit, G. M. 2005, Rev. Mod. Phys., 77, 207

Wen, Z. L., \& Han, J. L. 2015, ApJ, 807, 178

Wen, Z. L., Han, J. L., \& Yang, F. 2018, MNRAS, 475, 343

Wenger, M., Ochsenbein, F., Egret, D., et al. 2000, A\&AS, 143, 9 


\section{Appendix A: Characterization of the SPT-SZ filter transfer function}

The SPT-SZ filter transfer function is provided with the public data for each SPT frequency. We tested it by comparing point source fluxes extracted from the public data (using the provided transfer function) and the fluxes published for the same sources by the SPT collaboration. In practice, we adopted the positions of the point sources in the 2013 catalog (Mocanu et al. 2013) and extracted their flux using a single frequency matched filter applied to the public data. We used the SPT-SZ frequency responses given in Fig. 10 of Chown et al. (2018) (long-dashed lines). The results are presented in Fig. A.1 for the three SPT channels.

We restricted the extraction to sources with a published SPT flux above $1 \mathrm{mJy}$. The left-hand column shows our extracted flux as a function of the published SPT flux for the 95 (top figure), 150 (middle), and 220 (bottom) GHz channels. There is global agreement between the two. The right-hand column shows the ratio of the two fluxes versus the SPT flux. Blue diamonds are weighted averages of the individual measurements (red dots). We see that our fluxes are, in fact, systematically overestimated, in particular for flux $S$ above $50 \mathrm{mJy}$ (indicated by the vertical blue dotted line) for the three SPT frequencies. The overestimation is about $5 \%$ with respect to the published values. For fluxes below $50 \mathrm{mJy}$, there is no significant overestimation in the $95 \mathrm{GHz}$ channel, but there remains an overestimation for the 150 and $220 \mathrm{GHz}$ channels, although less significant than for point sources with fluxes above $50 \mathrm{mJy}$.
To investigate the origin of this effect, we stacked the SPTSZ maps at the locations of the bright $(S>50 \mathrm{mJy})$ and faint $(S<50 \mathrm{mJy})$ point sources separately. We then compared the two stacks to the beam convolved by the filter transfer function. Figure A. 2 presents the results for the $150 \mathrm{GHz}$ sources. The lefthand column gives the two stacks $(S<50 \mathrm{mJy}$ top, $S>50 \mathrm{mJy}$ bottom). The middle column shows the beam convolved by the filter transfer function (duplicated top and bottom). The right-hand column shows the difference between the first two columns. The stack of the faint sources displays the same pattern as the beam convolved with the filter transfer function, but the stack of the bright sources does not. This result confirms that the beam convolved by the filter transfer function does not correctly model the shape of the bright $(S>50 \mathrm{mJy})$ point sources in the data. A similar analysis of the $95 \mathrm{GHz}$ and $220 \mathrm{GHz}$ channels leads to the same conclusion, with different patterns in the stacks of point sources with a flux above and below $50 \mathrm{mJy}$ : The stacks of sources with fluxes below $50 \mathrm{mJy}$ show a pattern identical to the beams convolved by the filter transfer functions, while the stacks of sources with fluxes above $50 \mathrm{mJy}$ do not. This result is expected because the regions surrounding the bright $(S>50 \mathrm{mJy})$ point sources were excluded from the fitting of the time stream data (see Sect. 4.1.1 of Chown et al. 2018), so the filter transfer functions are not expected to model these regions of the maps.

The fact that bright sources are not well modeled by the beam convolved by the filter transfer function is the most probable explanation for the overestimation of the SZ flux of bright clusters $\left(Y^{0.75} \mathrm{SPT}>2 \times 10^{-4} \operatorname{arcmin}^{2}\right)$ in the SPT-SZ data with respect to the published SPT values. This issue is described in Appendix B and illustrated in Fig. B.3. 

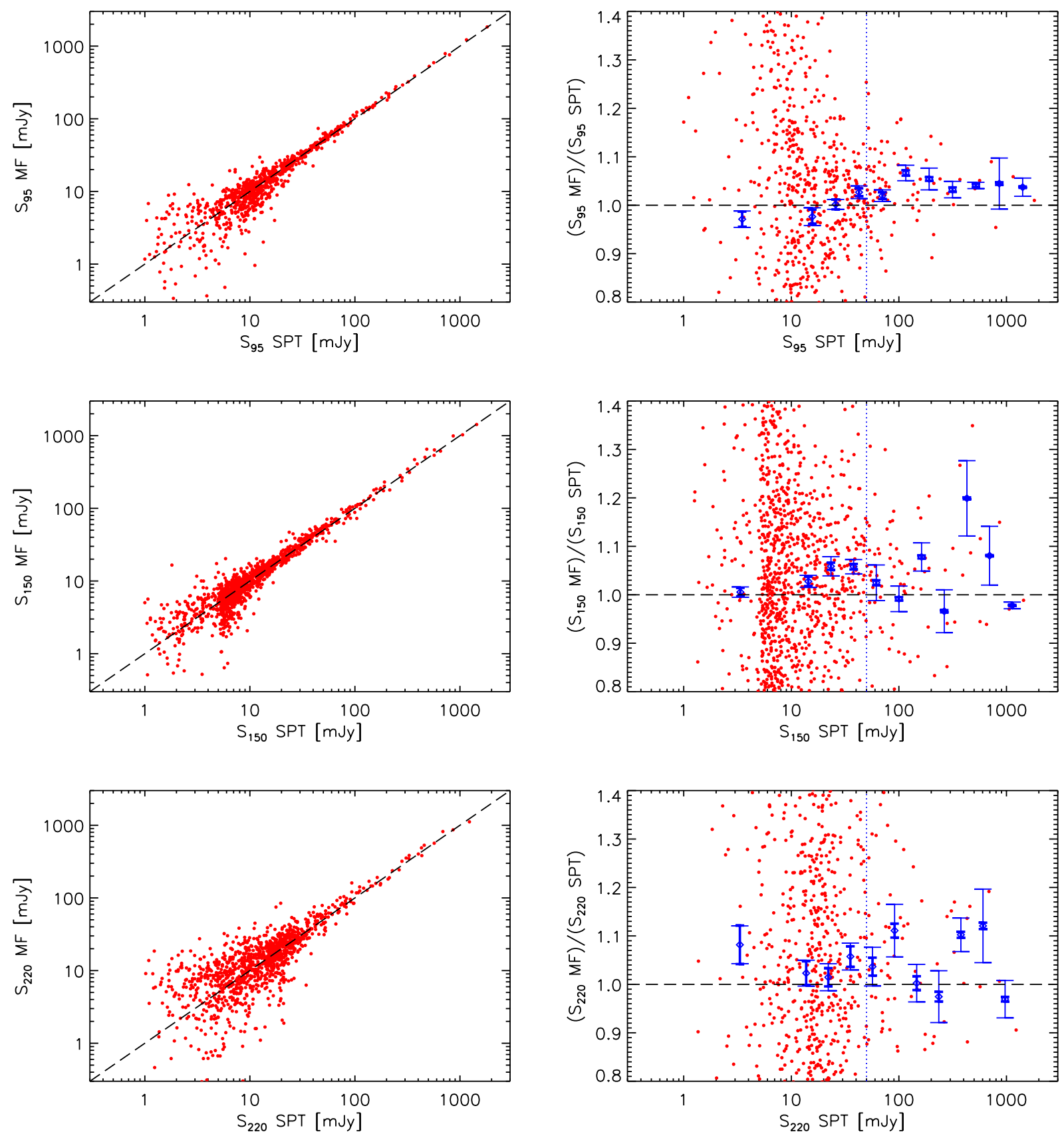

Fig. A.1. Left column: our single frequency matched filter flux versus published SPT flux for the point sources published in Mocanu et al. (2013) from $95 \mathrm{GHz}$ (top) to $220 \mathrm{GHz}$ (bottom). Red dots are individual point sources. There is overall agreement between our recovered fluxes and the values published by the SPT collaboration. Right column: zoom-in on the ratio between the two flux measurements as a function of the SPT flux. Blue diamonds are weighted averages. Thick bars display 68\% statistical errors, and thin bars show 68\% errors obtained by the bootstrap method. Despite the global agreement in the log-log plane shown in the left column, the ratio is significantly greater than unity, in particular at a large flux $(S>50 \mathrm{mJy})$ for the three SPT frequencies. We indicate this $50 \mathrm{mJy}$ limit by the vertical blue dotted lines in the three panels. 


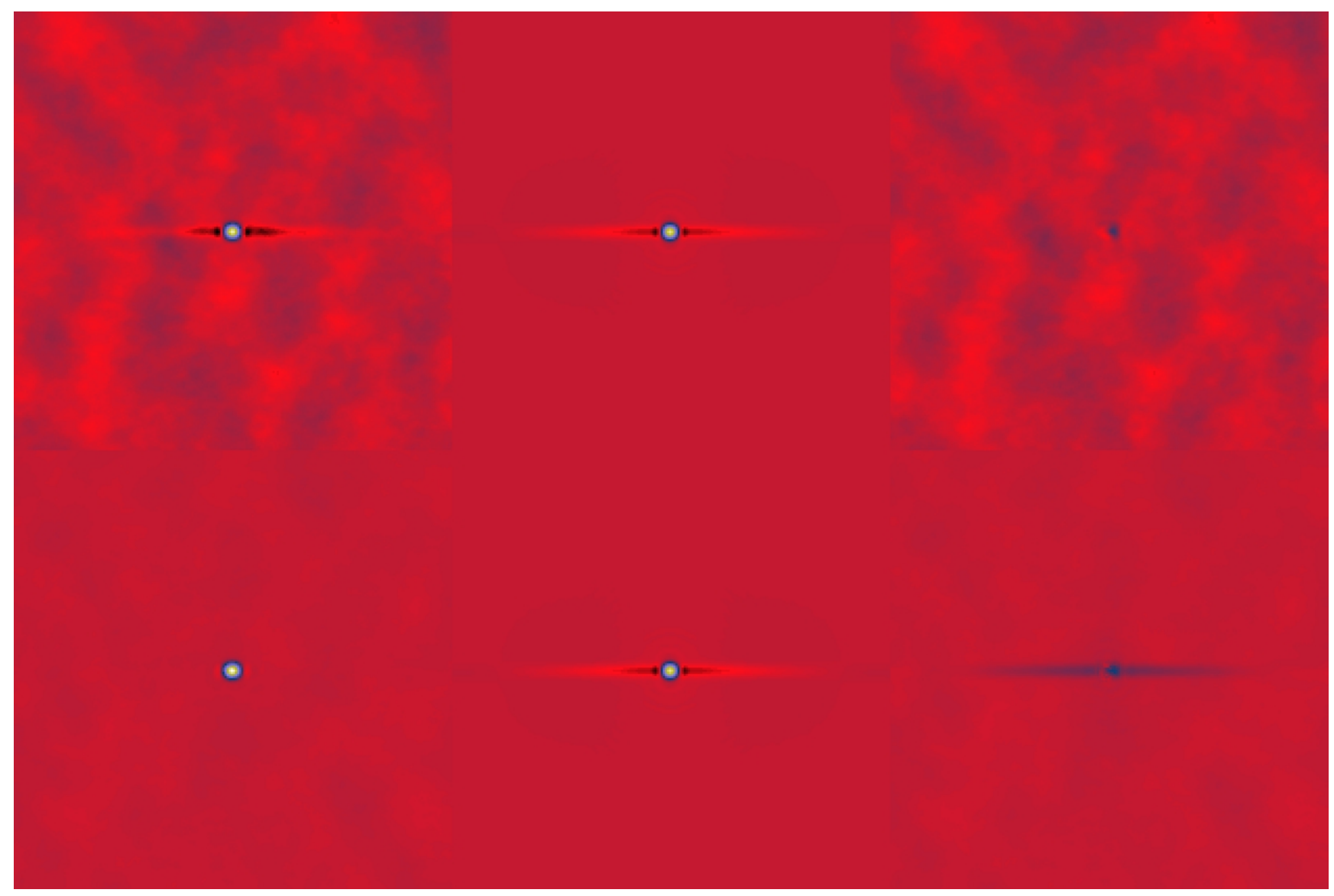

Fig. A.2. Stacks of the $150 \mathrm{GHz}$ point sources of Mocanu et al. (2013) from the public SPT-SZ data in equatorial coordinates (a horizontal line lies at constant declination). The six square panels are $1.5 \mathrm{deg}$ on a side. Top left: stack of point sources with published SPT flux below $50 \mathrm{mJy}$, normalized to the total flux of the stack computed from SPT published values. Top middle: Gaussian beam $(F W H M=1.75$ arcmin $)$ convolved with the filter transfer function. Top right: difference between top left and top middle images. The residuals are small. Bottom left: stack of point sources with published SPT flux above $50 \mathrm{mJy}$, normalized to the total flux of the stack computed from published SPT values. Bottom middle: identical to top middle, i.e., Gaussian beam $(F W H M=1.75 \mathrm{arcmin})$ convolved with the filter transfer function. Bottom right: difference between bottom left and bottom middle images. The residuals are significant. In particular, the stack does not show the negative tails before and after the central maximum in right ascension, which can be seen in black in the middle image. This difference in the patterns can be seen in the difference image as the horizontal trail.

\section{Appendix B: SPT-SZ photometry}

We re-extracted the flux and size of the SPT-SZ clusters, fixing the position to the coordinates provided in the SPT cluster catalog (Bleem et al. 2015a). The goal was to check the consistency between our photometry (i.e., flux and size estimation) and the photometry from the SPT collaboration. We adopted the cluster modeling of the SPT collaboration: $\beta$ profile of size $\theta_{c}$ ranging from 0.25 to 3 arcmin in steps of 0.25 and $\beta=1$ fixed. We allowed the filter size to vary in the aforementioned range and, for each cluster, we kept the size that maximizes the signalto-noise. We then extracted the cluster flux for this specific size.

Figure B.1 compares our extracted signal-to-noise (maximum across filter scales) to the signal-to-noise published by the SPT collaboration, $\xi$. The left-hand panel shows that our $\mathrm{S} / \mathrm{N}$ is in global agreement with the published SPT signal-tonoise, although it is systematically lower. The right-hand panel shows the ratio of the two signal-to-noise values. Our $\mathrm{S} / \mathrm{N}$ is, on average, 0.9 times $\xi$ for $\xi>5$. The SPT public maps (Chown et al. 2018) are based on the same 2008-2011 observation cuts as Mocanu et al. (2019), except for the $150 \mathrm{GHz}$ field based on the observation cuts from Story et al. (2013). Thus, they are shallower than the maps used in Bleem et al. (2015a) (see Table 1 therein). We suspect that the $\mathrm{S} / \mathrm{N}$ bias to lower values is due to the difference in map depths. We would expect this bias to disappear if the MMFs were applied to the same SPT data as used in Bleem et al. (2015a).

Figure B. 2 compares our recovered blind size to the size published by the SPT collaboration. Each histogram corresponds to clusters with the same size $\theta_{c}$ determined by the SPT collaboration. Our recovered sizes (red histograms) show no significant deviation from the size published by the SPT collaboration (vertical black line). The thick dashed vertical blue line shows the median of our recovered sizes, which is in good agreement with the SPT size.

Figure B. 3 compares our blind SZ flux to the flux published by the SPT collaboration. The left panel shows that there is good agreement between the two flux values at a low flux $\left(Y^{0.75} \mathrm{SPT}<\right.$ $2 \times 10^{-4} \operatorname{arcmin}^{2}$ ), but that our blind flux is systematically overestimated at a high flux $\left(Y^{0.75} \mathrm{SPT}>2 \times 10^{-4} \operatorname{arcmin}^{2}\right)$. The right panel shows the ratio of the two flux values: The overestimation is about $10 \%$ for $Y^{0.75} \mathrm{SPT}>2 \times 10^{-4} \mathrm{arcmin}^{2}$. Since the cluster size is correctly estimated, as shown in Fig. B.2, we attribute this overestimation to the inadequate modeling of bright sources by the filter transfer function provided by the SPT collaboration, as discussed in Appendix A. 

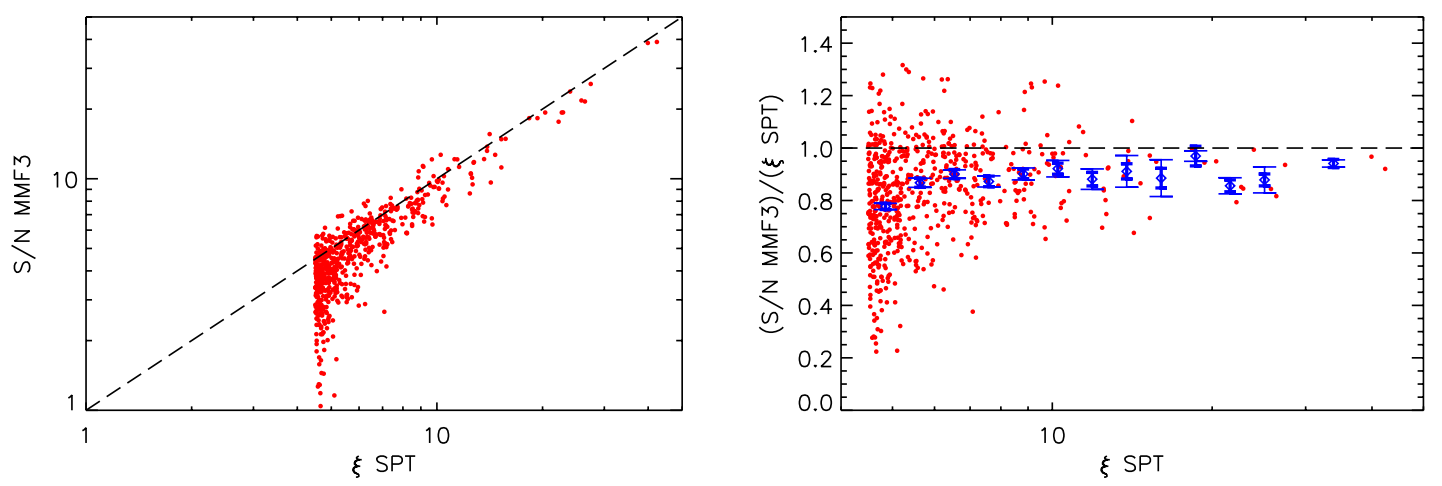

Fig. B.1. Left: S/N of the SPT clusters extracted from the public SPT data with our modified MMF3 algorithm as a function of the signal-to-noise, $\xi$, published by the SPT collaboration (Bleem et al. 2015a). Despite overall agreement, our S/N is systematically lower than the signal-to-noise published by the SPT collaboration. Right: ratio of the two signal-to-noise values as a function of the signal-to-noise published by the SPT collaboration. Our S/N is, on average, 0.9 times $\xi$ for $\xi>5$. Red dots are individual clusters. Blue diamonds are weighted averages. Thick error bars display $68 \%$ statistical errors, and thin error bars show $68 \%$ errors obtained by the bootstrap method.
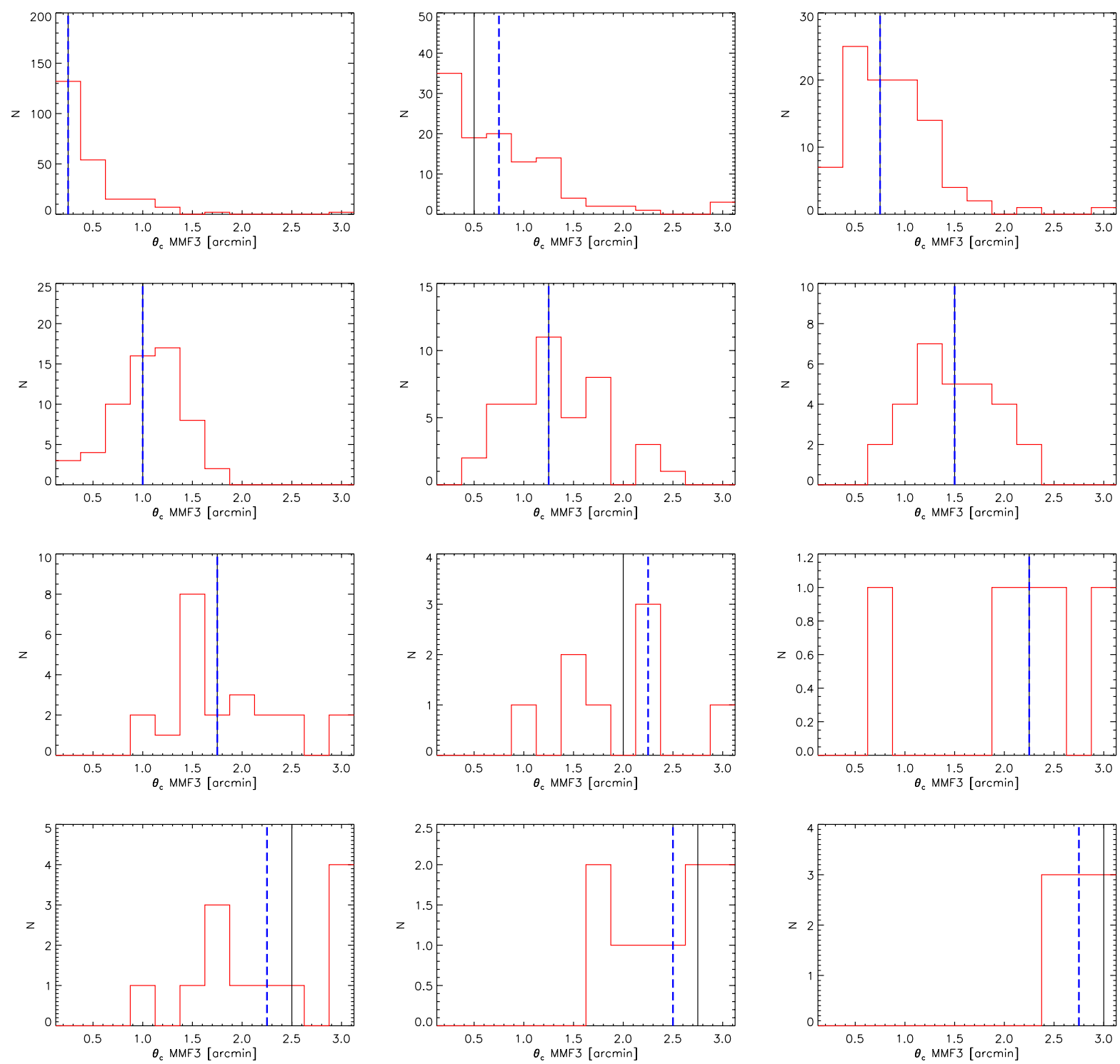

Fig. B.2. Histograms of cluster size as recovered by our algorithm. Each histogram corresponds to clusters with the same size $\theta_{c}$ determined by the SPT collaboration. The vertical black line displays the size from the SPT collaboration increasing from 0.25 to 3 arcmin in steps of 0.25 arcmin, from left to right and from top to bottom. The red histogram shows the distribution of the size recovered by our algorithm and the thick dashed vertical blue line is the median value of our recovered values. Our recovered sizes are in good agreement with the sizes published by the SPT collaboration. 

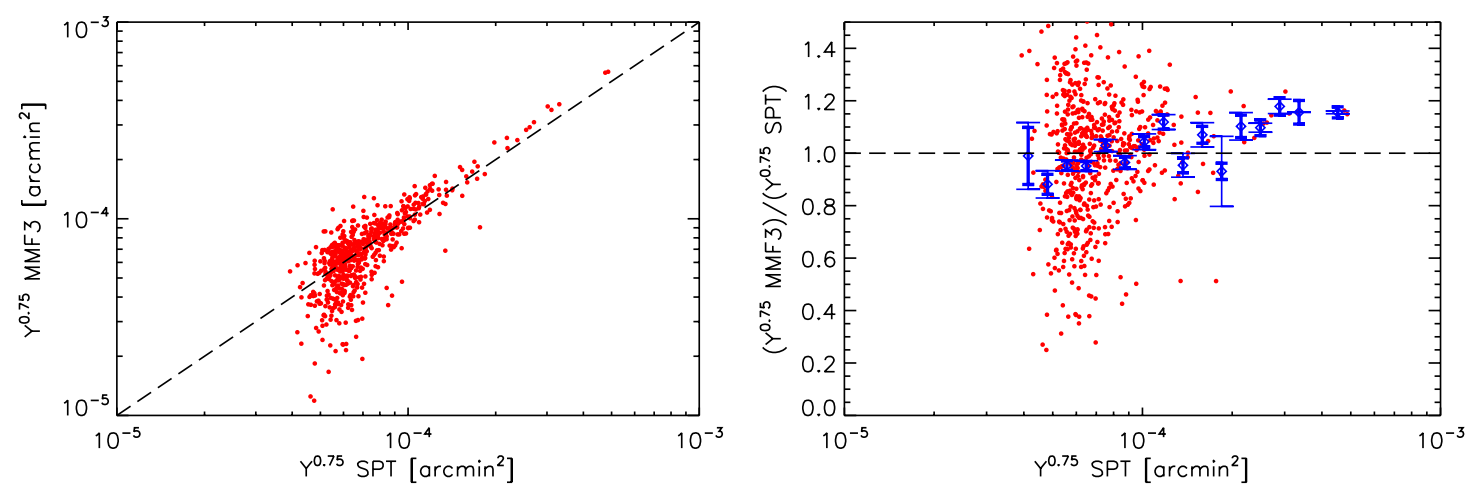

Fig. B.3. Left: blind SZ flux of the SPT clusters extracted from the public SPT data with our modified MMF3 algorithm as a function of the SZ flux $Y^{0.75}$ published by the SPT collaboration (Bleem et al. 2015a). There is good agreement at low flux, but our flux is systematically overestimated at high values $\left(Y^{0.75} \mathrm{SPT}>2 \times 10^{-4} \mathrm{arcmin}^{2}\right)$. Right: ratio of the two flux values as a function of the flux published by the SPT collaboration. Our flux is in good agreement for $Y^{0.75} \mathrm{SPT}<2 \times 10^{-4} \operatorname{arcmin}^{2}$, but it is on average 1.1 times $Y^{0.75} \mathrm{SPT}$ for $Y^{0.75} \mathrm{SPT}>2 \times 10^{-4}$ arcmin ${ }^{2}$. Red dots are individual clusters. Blue diamonds are weighted averages. Thick error bars display $68 \%$ statistical errors, and thin error bars show $68 \%$ errors obtained by the bootstrap method.

\section{Appendix C: Planck photometry}
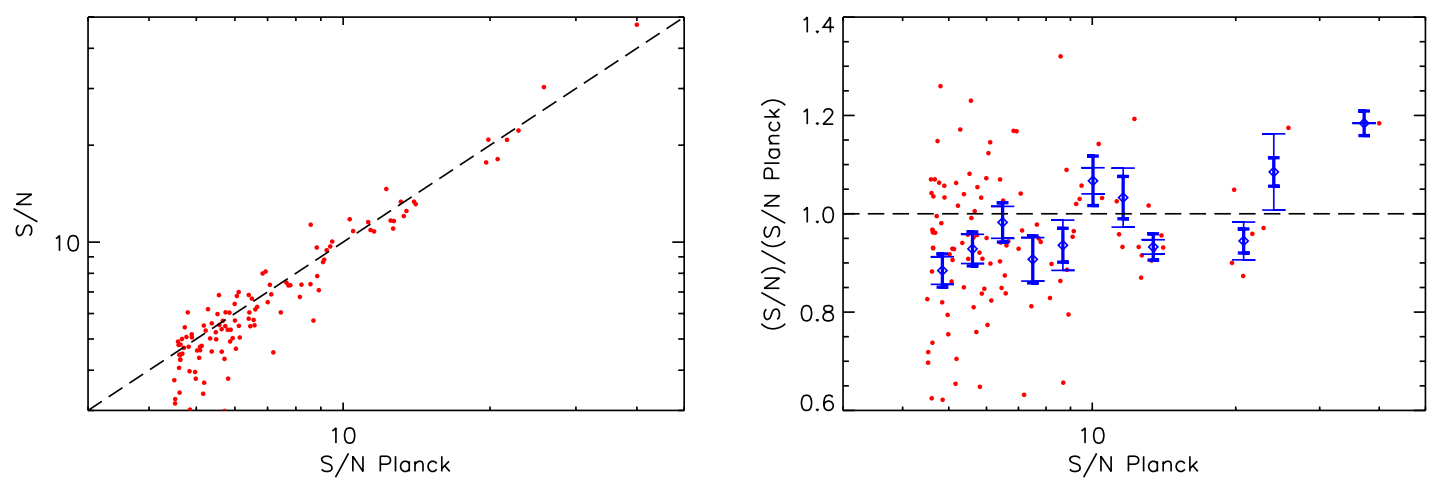

Fig. C.1. Left: $\mathrm{S} / \mathrm{N}$ of the Planck clusters extracted from the public Planck data as a function of the signal-to-noise $S / N$ Planck published by the Planck collaboration (Planck Collaboration XXVII 2016a). There is overall agreement, but also a large scatter between the two measurements due to the upgraded pixel size, the change of coordinate system, and the change in the point-source masking procedure. Right: ratio of the two signal-to-noise values as a function of the signal-to-noise published by the Planck collaboration. Red dots are individual clusters. Blue diamonds are weighted averages. Thick error bars display $68 \%$ statistical errors, and thin error bars show $68 \%$ errors obtained by the bootstrap method.

We re-extracted the flux and size of Planck clusters, fixing the position to the coordinates provided in the PSZ2 catalog (Planck Collaboration XXVII 2016a) in order to check the consistency between our photometry (i.e., flux and size estimation) and the photometry published by the Planck collaboration. We restricted the PSZ2 cluster catalog to MMF3-only clusters, since our extraction method is derived from it, and we solely focused on the clusters in the SPT footprint. The differences are not expected to be negligible, because we worked with upgraded Planck maps $\left(N_{\text {side }}=8192\right)$ instead of native Planck maps $\left(N_{\text {side }}=2048\right)$ and we changed the coordinate system from Galactic to equatorial to match the SPT-SZ public data. Although the all-sky maps that we used are the same as the all-sky maps used by the Planck collaboration and thus carry the same information, our $10 \times 10$ deg tangential maps around the PSZ2 clusters are different due to the different pixel size and map orientation. This changes the estimation of the noise power spectrum, $\boldsymbol{P}(\boldsymbol{k})$. In addition, we did not use the refined point source masking procedure used by the Planck collaboration (Sect. 3.1 of Planck Collaboration XXVII 2016a), but we detected the point sources above $S / N>8$ with single frequency matched filters in indi- vidual channel maps and masked them with a ten-arcmin radius disk, which is simpler and computationally faster.

Figure C.1 shows the results for signal-to-noise. The agreement between our $\mathrm{S} / \mathrm{N}$ and the $\mathrm{S} / \mathrm{N}$ published by the Planck collaboration is good, but there is a large scatter due to the upgrades to the pixel size, the change of coordinates, and the difference in the point source masking procedures. Planck blind sizes and fluxes were measured at the location of the maximum of the degeneracy contours provided by the PSZ2 cluster catalog. They were compared to the sizes and fluxes provided by our filter at the maximum of the $\mathrm{S} / \mathrm{N}$. The results are presented in Fig. C.2 for the sizes. The blind sizes are also in global good agreement, but some clusters show large deviations as displayed in the left panel. They correspond to clusters detected at low S/N for which the size determination is uncertain, as shown in the right-hand panel. These clusters are marked as red crosses in both panels.

Figure C.3 shows results for the flux measurements. As for the sizes, the global agreement is good except for some specific clusters. They correspond to clusters that we marked with the red crosses in Fig. C.2. Due to the low $\mathrm{S} / \mathrm{N}$ of some detections, the size was poorly determined, which directly translates into a bad recovery of the integrated flux. 

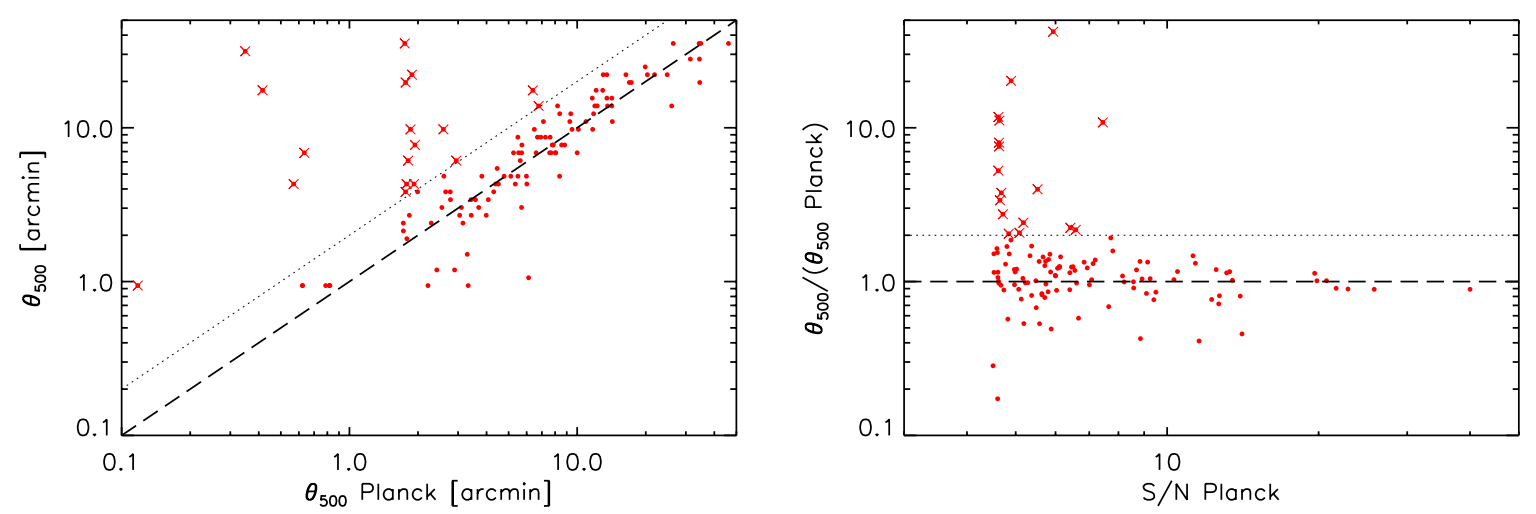

Fig. C.2. Left: blind size $\theta_{500}$ of the Planck clusters extracted from the public Planck data as a function of the blind size $\theta_{500}$ Planck published by the Planck collaboration (Planck Collaboration XXVII 2016a). Red dots are individual clusters. There is overall agreement, but some clusters show deviations larger than a factor of two. They are marked with a red cross. The dotted black line delineates the deviation by a factor of two. Right: ratio of the two size values as a function of the $\mathrm{S} / \mathrm{N}$ published by the Planck collaboration. The deviating clusters are mainly located at the lower $\mathrm{S} / \mathrm{N}$, for which the blind size estimation is uncertain.
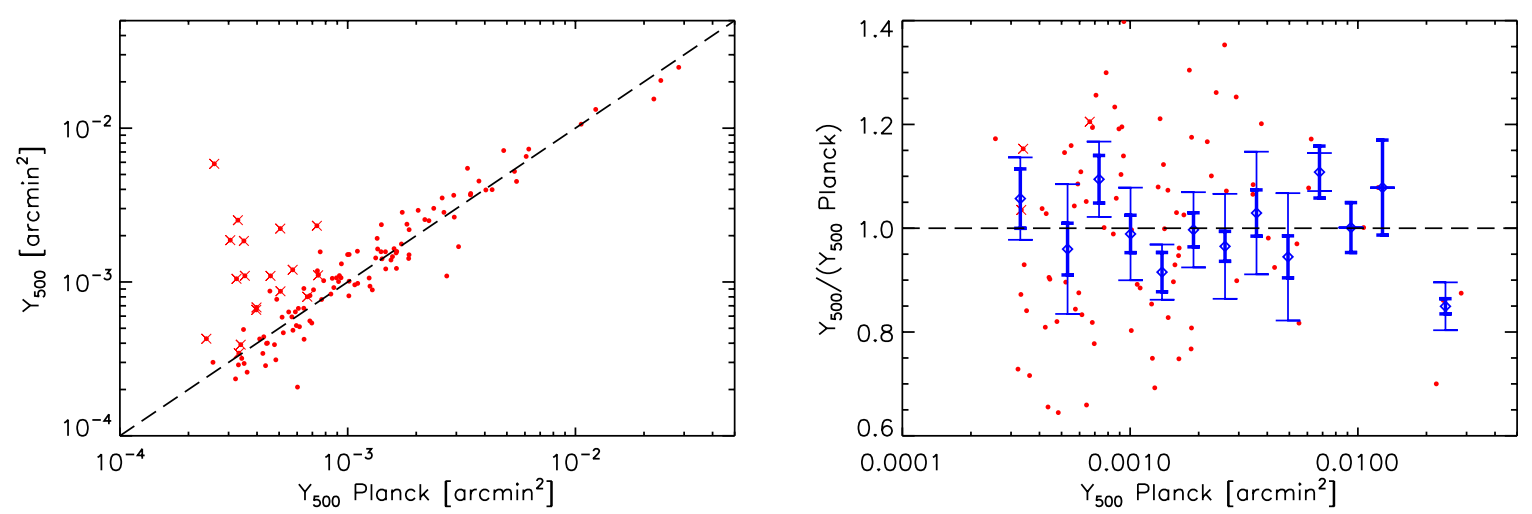

Fig. C.3. Left: blind flux $Y_{500}$ of the Planck clusters extracted from the public Planck data as a function of the blind flux $Y_{500}$ Planck published by the Planck collaboration (Planck Collaboration XXVII 2016a). Red dots are individual clusters. There is overall agreement, but some clusters show deviations from the equality line. They correspond to clusters having blind sizes deviating by more than a factor of two from the values published by the Planck collaboration and are marked as red crosses (see also Fig. C.2). Right: ratio of the two flux values as a function of the flux published by the Planck collaboration. The ratio is consistent with unity. Blue diamonds are weighted averages. Thick error bars display $68 \%$ statistical errors, and thin error bars show $68 \%$ errors obtained by the bootstrap method.

\section{Appendix D: Comparison of masses extracted by the joint matched filter and masses published by the SPT and Planck collaborations}

In this appendix, we study outliers in Fig. 5 and 8 (Sect. D.1) and we investigate the bias in the recovered joint mass with respect to the published mass for PSZ2 clusters (Appendix D.2).

\section{D.1. Outliers in mass}

We mark the four clear outliers of Figs. 5 and 8 with blue symbols. In Fig. D.1, the circle marks SPT-CLJ2313-4243 $(z=0.0564$, also PSZ2 G348.46-64.83), the downward triangle SPT-CLJ0439-5330 $(z=0.43)$, and the upward triangle SPT-CLJ0431-6126 ( $z=0.0577$, also PSZ2 G272.08-40.16). In Fig. D.2, the square marks PSZ2 G271.53-56.57 $(z=0.3)$.

We visually inspected the filtered maps of these four clusters and found that SPT-CLJ0431-6126 and PSZ2 G271.5356.57 are located close to masked point sources, which may have contaminated their fluxes and thus their masses as well. We did not notice any problem in the filtered maps of the two other outliers. SPT-CLJ2313-4243 and SPT-CLJ0431-6126 are marked as outliers in Fig. D.1, but they are actually not outliers in Fig. D.2. This suggests that the published SPT and Planck masses do not match for these two clusters. Indeed, this is con-

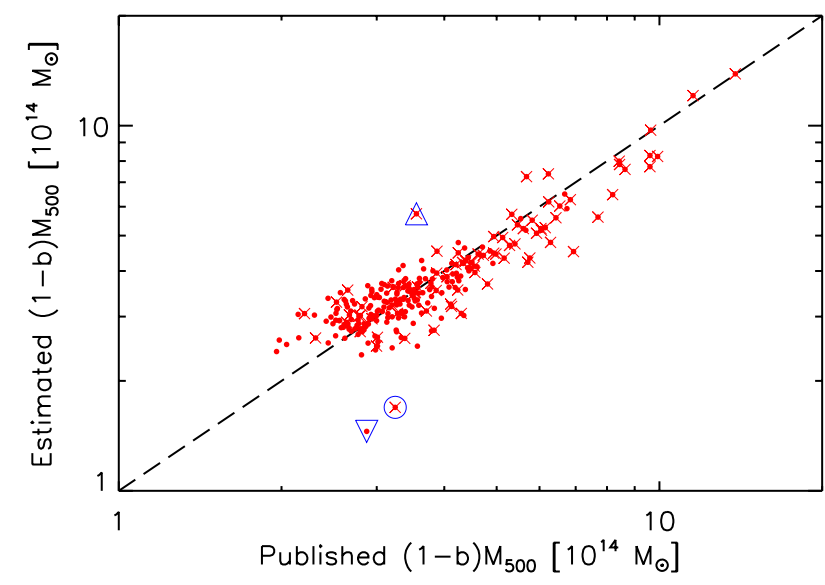

Fig. D.1. Extracted masses versus published SPT-SZ masses for the joint detections matched to SPT-SZ clusters. This figure is the same as Fig. 5, but we additionally marked the clusters that are both matched to SPT-SZ and PSZ2 clusters with red crosses. We also marked three outliers with a blue circle and upward and downward triangles.

firmed in Fig. D.3: SPT-CLJ2313-4243 is a clear outlier and SPT-CLJ0431-6126 is at the edge of the distribution. We further investigate the distance between the SPT-SZ and PSZ2 positions 


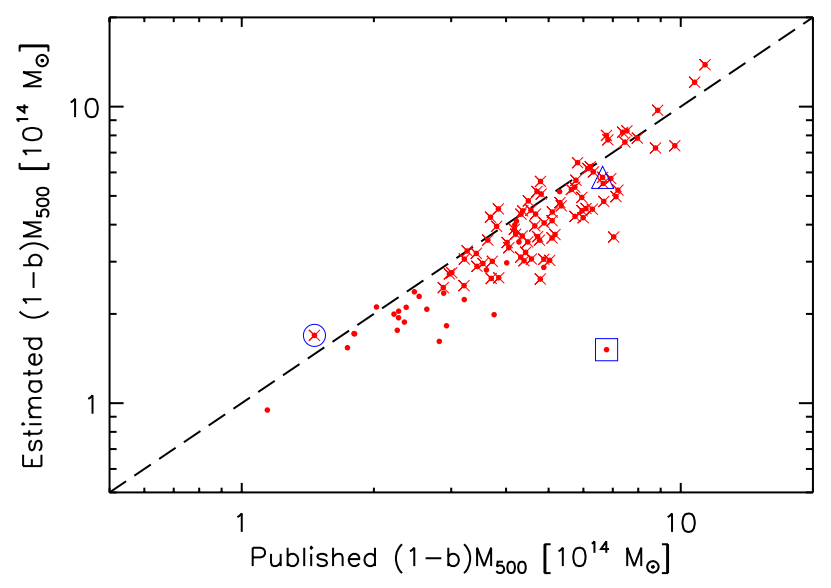

Fig. D.2. Extracted masses versus published PSZ2 masses for the joint detections matched to PSZ2 clusters. This figure is the same as Fig. 8, but we additionally marked the clusters that are both matched to SPTSZ and PSZ2 clusters with red crosses as in Fig. D.1. We also marked the main outlier with a blue square. The blue circle and upward triangle, which are outliers in Fig. D.1, are not outliers in this figure.

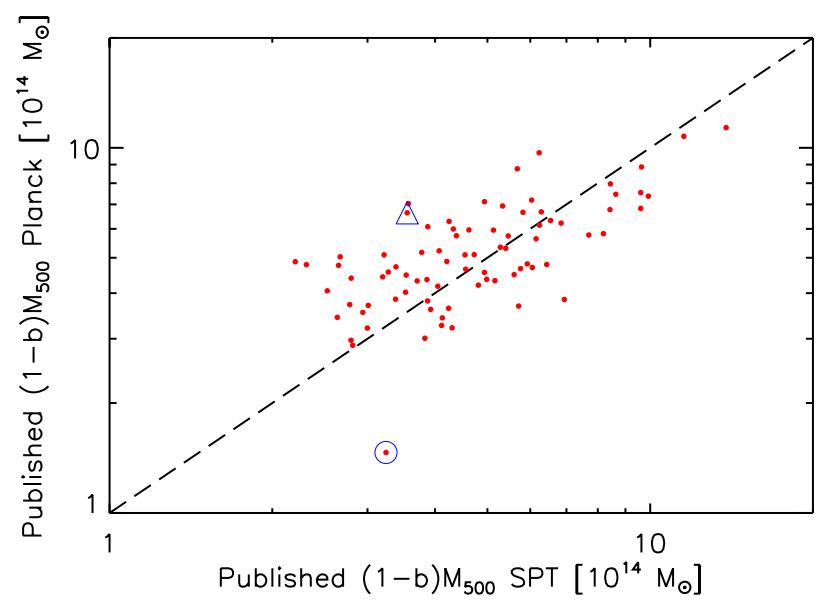

Fig. D.3. Published PSZ2 masses versus published SPT-SZ masses for the clusters that are both in the SPT-SZ and PSZ2 published catalogs. The cluster marked with a blue circle is an outlier.

for these two clusters as a possible explanation for them being outliers (see Sect. 3.2 of Tarrío et al. 2019), but we found the two offsets to be in the overall distribution of the offsets of the detections matching both SPT-SZ and PSZ2 clusters.

In summary, the outliers may originate from flux contamination due to close by point sources and/or to a miss-match of the masses already present in the published SPT-SZ and PSZ2 catalogs. This mass miss-match in the original catalogs can also be due to point source contamination or specific issues from the datasets (e.g., flux estimation of nearby clusters such

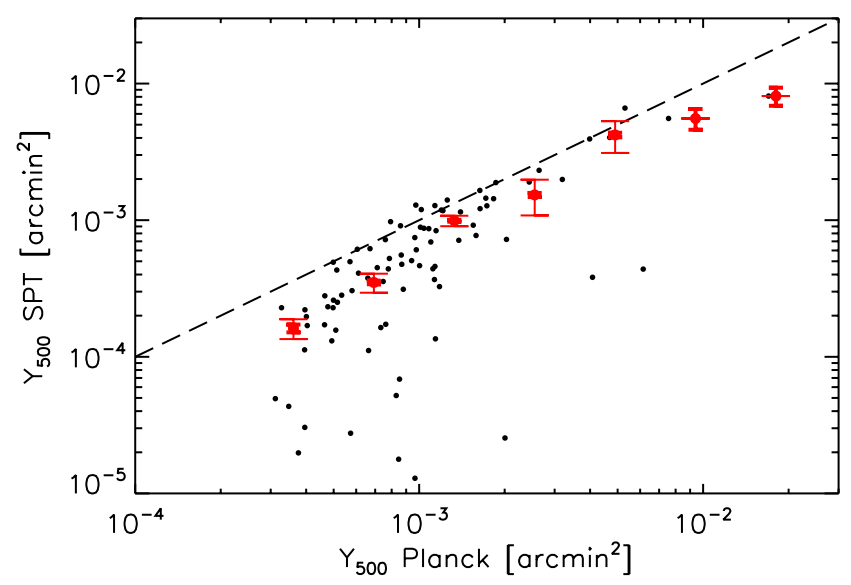

Fig. D.4. SPT fluxes of PSZ2 clusters as a function of PSZ2 flux. This is the same figure as Fig. 2, except that we used the XMM-Newton prior to fixing the filter size, instead of the blind PSZ2 size, for both the Planck fluxes ( $x$-axis) and SPT fluxes ( $y$-axis).

as SPT-CLJ2313-4243 and SPT-CLJ0431-6126 in the SPT data may be difficult).

\section{D.2. Joint versus published mass for PSZ2 clusters}

Figure 8 shows a small underestimation of the joint mass with respect to the published PSZ2 mass. We investigate if this trend is visible for detections matching PSZ2-only or for detections matching both SPT-SZ and PSZ2 clusters. In Fig. D.2, we mark detections matching both SPT-SZ and PSZ2 clusters with red crosses: They show the same trend as detections matching PSZ2only clusters. We also mark the SPT-SZ and PSZ2 clusters in Fig. D.1 with red crosses: They also show a small underestimation of the joint mass with respect to the published SPT-SZ mass. Detections matching SPT-SZ only do not show a bias.

This underestimation of the joint mass with respect to the published mass is very likely due to the underestimation of the SPT flux with respect to Planck flux when using the XMMNewton prior to determining the filter size instead of the blind size. The joint mass is determined using the XMM-Newton prior. When using this prior to estimate PSZ2 cluster masses in the SPT-SZ data, the SPT flux are systematically smaller than the Planck flux as shown in Fig. D.4. The consequence of a smaller SPT flux is a smaller joint flux and thus a smaller mass.

In all the figures of Appendix D, the published SPT masses were recalibrated by 0.8 to account for our mass definition.

\section{Appendix E: Names of the missed SPT and PSZ2 clusters}

In Tables E.1 and E.2, we provide the names of the missed SPT and PSZ2 clusters studied in Sect. 6.3. 
Table E.1. 101 SPT clusters missed in the PSZSPT catalog.

\begin{tabular}{llllll}
\hline \hline SPT-CLJ0001-5440 & SPT-CLJ0002-5557 & SPT-CLJ0011-4614 & SPT-CLJ0013-5714 & SPT-CLJ0015-6000 & SPT-CLJ0021-4902 \\
SPT-CLJ0022-4144 & SPT-CLJ0052-4551 & SPT-CLJ0108-4659 & SPT-CLJ0119-5919 & SPT-CLJ0135-5902 & SPT-CLJ0139-5204 \\
SPT-CLJ0144-4157 & SPT-CLJ0148-4518 & SPT-CLJ0201-6051 & SPT-CLJ0202-5401 & SPT-CLJ0216-5730 & SPT-CLJ0221-4446 \\
SPT-CLJ0231-4427 & SPT-CLJ0242-4150 & SPT-CLJ0257-5732 & SPT-CLJ0257-4817 & SPT-CLJ0313-5334 & SPT-CLJ0337-6300 \\
SPT-CLJ0337-6207 & SPT-CLJ0341-6143 & SPT-CLJ0344-5518 & SPT-CLJ0353-5043 & SPT-CLJ0354-5151 & SPT-CLJ0412-5106 \\
SPT-CLJ0418-4552 & SPT-CLJ0421-4845 & SPT-CLJ0422-4608 & SPT-CLJ0428-6049 & SPT-CLJ0430-6251 & SPT-CLJ0433-5630 \\
SPT-CLJ0444-5603 & SPT-CLJ0444-4352 & SPT-CLJ0454-4211 & SPT-CLJ0455-4159 & SPT-CLJ0456-4906 & SPT-CLJ0459-4947 \\
SPT-CLJ0500-5116 & SPT-CLJ0512-5139 & SPT-CLJ0516-5755 & SPT-CLJ0522-5026 & SPT-CLJ0528-4417 & SPT-CLJ0529-6051 \\
SPT-CLJ0533-5005 & SPT-CLJ0535-5956 & SPT-CLJ0539-6013 & SPT-CLJ0540-5744 & SPT-CLJ0550-6358 & SPT-CLJ0559-6022 \\
SPT-CLJ0611-4724 & SPT-CLJ0617-5507 & SPT-CLJ0622-4645 & SPT-CLJ0625-4330 & SPT-CLJ0637-6112 & SPT-CLJ0637-4327 \\
SPT-CLJ0643-4535 & SPT-CLJ0643-5056 & SPT-CLJ0649-4510 & SPT-CLJ0650-4503 & SPT-CLJ2011-5725 & SPT-CLJ2020-6314 \\
SPT-CLJ2026-4513 & SPT-CLJ2030-5638 & SPT-CLJ2040-5342 & SPT-CLJ2040-4451 & SPT-CLJ2043-5035 & SPT-CLJ2051-6256 \\
SPT-CLJ2056-5459 & SPT-CLJ2058-5608 & SPT-CLJ2101-6123 & SPT-CLJ2106-4421 & SPT-CLJ2108-4445 & SPT-CLJ2109-5040 \\
SPT-CLJ2111-5339 & SPT-CLJ2120-4728 & SPT-CLJ2136-4704 & SPT-CLJ2140-5727 & SPT-CLJ2143-5509 & SPT-CLJ2145-4348 \\
SPT-CLJ2152-5633 & SPT-CLJ2155-5224 & SPT-CLJ2155-6048 & SPT-CLJ2158-5451 & SPT-CLJ2203-5047 & SPT-CLJ2205-5927 \\
SPT-CLJ2232-6151 & SPT-CLJ2235-4416 & SPT-CLJ2254-5805 & SPT-CLJ2259-5431 & SPT-CLJ2301-5546 & SPT-CLJ2308-4834 \\
SPT-CLJ2311-4203 & SPT-CLJ2311-5820 & SPT-CLJ2321-5419 & SPT-CLJ2352-5846 & SPT-CLJ2353-5512 & \\
\hline
\end{tabular}

Table E.2. 11 PSZ2 clusters missed in the PSZSPT catalog.

\begin{tabular}{llllll}
\hline \hline PSZ2 G265.21-24.83 & PSZ2 G341.09-33.15 & PSZ2 G331.96-45.74 & PSZ2 G255.52-35.66 & PSZ2 G327.66-54.26 & PSZ2 G336.01-51.27 \\
PSZ2 G269.36-47.20 & PSZ2 G319.64-65.11 & PSZ2 G265.60-46.87 & PSZ2 G299.68-60.21 & PSZ2 G279.51-44.85 & \\
\hline
\end{tabular}

\section{Appendix F: Description of the PSZSPT catalog}

The PSZSPT catalog contains 419 detections. For each detection, we provide the following fields, partially shown in Table F.1. A complete version of the catalog is provided in electronic format at the CDS.

- NAME: Name of the candidate, PSZSPT Jxxxx+yyyy

- RAJ2000: Right ascension (J2000) in degrees

- DEJ2000: Declination (J2000) in degrees

- GLON: Galactic longitude in degrees

- GLAT: Galactic latitude in degrees

- SNR: Signal-to-noise ratio obtained with the best filter size

- RANK: Rank of the candidate $(0=$ unidentified; 1 = identified; 2 = possibly identified; $3=$ multiple detection)

- Z: Redshift of the candidate

- Z_REF: Origin of the redshift

- M500: Estimated cluster mass in solar masses

- M500_INF: Lower bound of $68 \%$ confidence interval on the estimated cluster mass in solar masses

- M500_SUP: Upper bound of 68\% confidence interval on the estimated cluster mass in solar masses

- SPT: Name of the SPT (Bleem et al. 2015a) cluster matched to the candidate
- PSZ2: Name of the Planck PSZ2 (Planck Collaboration XXVII 2016a) cluster matched to the candidate

- MCSZ: Name of the MCSZ (https://www . galaxyclusterdb.eu/) cluster matched to the candidate

- MCXC: Name of the MCXC (Piffaretti et al. 2011) cluster matched to the candidate

- COMPRASS: Name of the ComPRASS (Tarrío et al. 2019) cluster matched to the candidate

- ABELL: Name of the Abell (Abell et al. 1989) cluster matched to the candidate

- BCS: Name of the BCS (Desai et al. 2012; Bleem et al. 2015b) cluster matched to the candidate

- MARD: Name of the MARD-Y3 (Klein et al. 2019) cluster matched to the candidate

- WHY: Name of the WHY (Wen et al. 2018) cluster matched to the candidate

- SIMBAD: Name of the SIMBAD counterpart found in a 20 arcmin radius disk around the candidate

- NED: Name of the NED counterpart found in a 20 arcmin radius disk around the candidate.

- NOTES: Notes on specific candidates. 
Table F.1. Cluster candidates in the PSZSPT catalog.

\begin{tabular}{|c|c|c|c|c|c|c|c|c|c|c|c|}
\hline NAME & $\begin{array}{c}\text { RAJ2000 } \\
\text { [deg] }\end{array}$ & $\begin{array}{c}\text { DEJ2000 } \\
\text { [deg] }\end{array}$ & $\begin{array}{c}\text { GLON } \\
\text { [deg] }\end{array}$ & $\begin{array}{r}\text { GLAT } \\
{[\mathrm{deg}]}\end{array}$ & $S / N$ & RANK & $\mathrm{Z}$ & Z_REF & $\begin{array}{c}\text { M500 } \\
{\left[10^{14} M_{\odot}\right]}\end{array}$ & $\begin{array}{c}\text { M500_INF } \\
{\left[10^{14} M_{\odot}\right]}\end{array}$ & $\begin{array}{c}\text { M500_SUP } \\
{\left[10^{14} M_{\odot}\right]}\end{array}$ \\
\hline PSZSPT J0000-4356 & 0.061 & -43.949 & 331.122 & -70.270 & 5.19 & 1 & 1.000 & SPT & 2.61 & 2.23 & 2.97 \\
\hline PSZSPT J0000-5748 & 0.241 & -57.808 & 315.638 & -58.056 & 7.40 & 1 & 0.701 & SPT & 3.48 & 3.15 & 3.80 \\
\hline PSZSPT J0001-4843 & 0.292 & -48.718 & 323.868 & -66.315 & 6.65 & 1 & 0.300 & SPT & 3.17 & 2.81 & 3.51 \\
\hline PSZSPT J0003-5253 & 0.840 & -52.889 & 318.846 & -62.727 & 5.07 & 1 & 0.279 & WHY & 2.50 & 2.13 & 2.84 \\
\hline PSZSPT J0012-5352 & 3.073 & -53.872 & 315.381 & -62.299 & 6.15 & 1 & 0.330 & SPT & 2.82 & 2.47 & 3.15 \\
\hline PSZSPT J0013-4907 & 3.320 & -49.117 & 318.920 & -66.808 & 12.3 & 1 & 0.406 & SPT & 5.17 & 4.86 & 5.47 \\
\hline PSZSPT J0015-5303 & 3.861 & -53.064 & 314.969 & -63.213 & 5.10 & 0 & -1.00 & & -1.0 & -1.0 & -1.0 \\
\hline PSZSPT J0019-5527 & 4.819 & -55.452 & 312.372 & -61.081 & 7.02 & 1 & 0.755 & SPT & 3.39 & 3.06 & 3.71 \\
\hline PSZSPT J0025-4133 & 6.490 & -41.551 & 321.219 & -74.659 & 6.39 & 1 & 0.430 & SPT & 2.87 & 2.57 & 3.16 \\
\hline
\end{tabular}

\begin{tabular}{|c|c|c|c|c|}
\hline NAME & SPT & PSZ2 & MCSZ & MCXC \\
\hline PSZSPT J0000-4356 & SPT-CLJ0000-4356 & & SPT-CLJ0000-4356 & \\
\hline PSZSPT J0000-5748 & SPT-CLJ0000-5748 & & SPT-CLJ0000-5748 & \\
\hline PSZSPT J0001-4843 & SPT-CLJ0001-4842 & & SPT-CLJ0001-4842 & \\
\hline PSZSPT J0003-5253 & & & & \\
\hline PSZSPT J0012-5352 & SPT-CLJ0012-5352 & & SPT-CLJ0012-5352 & \\
\hline PSZSPT J0013-4907 & SPT-CLJ0013-4906 & & SPT-CLJ0013-4906 & \\
\hline PSZSPT J0014-4952 & SPT-CLJ0014-4952 & & SPT-CLJ0014-4952 & \\
\hline PSZSPT J0015-5303 & & & & \\
\hline PSZSPT J0019-5527 & SPT-CLJ0019-5527 & & SPT-CLJ0019-5527 & \\
\hline PSZSPT J0025-4133 & SPT-CLJ0025-4133 & & SPT-CLJ0025-4133 & \\
\hline
\end{tabular}

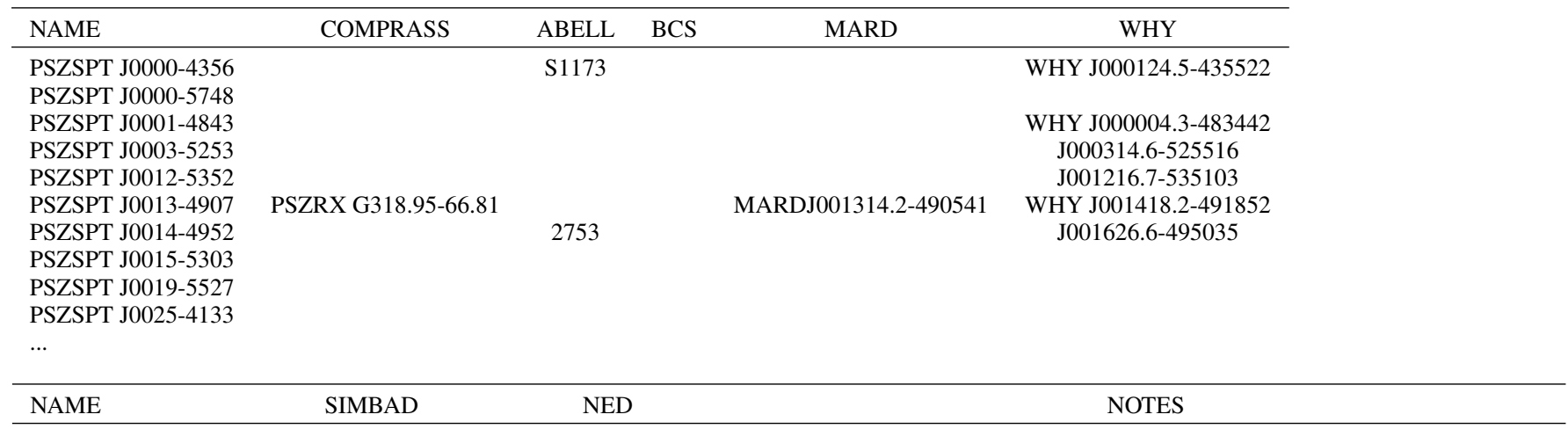

\begin{tabular}{|c|c|c|c|}
\hline NAME & SIMBAD & NED & NOTES \\
\hline $\begin{array}{l}\text { PSZSPT J0000-4356 } \\
\text { PSZSPT J0000-5748 } \\
\text { PSZSPT J0001-4843 } \\
\text { PSZSPT J0003-5253 } \\
\text { PSZSPT J0012-5352 } \\
\text { PSZSPT J0013-4907 } \\
\text { PSZSPT J0014-4952 } \\
\text { PSZSPT J0015-5303 } \\
\text { PSZSPT J0019-5527 } \\
\text { PSZSPT J0025-4133 } \\
\ldots\end{array}$ & SPT-CL J0013-5310 & SPT-CL J0013-5310 & SPT-CL J0013-5310 @ 17.4 arcmin is not associated to another PSZSPT detection \\
\hline
\end{tabular}

Notes. The different fields are described in the text of this appendix. This table is available in its entirety in a machine-readable format at the CDS. 